\title{
Iron and nickel atoms in cometary atmospheres even far from the Sun
}

\author{
J. Manfroid, D. Hutsemékers, and E. Jehin \\ STAR Institute, University of Liège \\ Allée du 6 Août 19c, B-4000 Liège Belgium
}

February 02, 2020

\begin{abstract}
In comets, iron and nickel are found in refractory dust particles or in metallic and sulfide grains ${ }^{1}$. No iron- or nickel-bearing molecules have been observed in the gaseous coma of comets $^{2}$. Iron and a few other heavy atoms such as copper and cobalt were only observed in two exceptional objects, the Great Comet of $1882^{3}$ and, almost a century later, C/1965 S1 (Ikeya-Seki) $^{4-9}$. These sungrazing comets approached the Sun so close that the refractory materials could sublimate. The relative abundance of nickel to iron was similar to the Sun and the meteorites ${ }^{7}$. More recently the presence of iron vapor was inferred from the properties of a faint tail in comet C/2006 P1 (McNaught) at perihelion ${ }^{10}$ but neither iron nor nickel was reported in the gaseous coma of comet 67P/Churyumov-Gerasimenko by the in situ Rosetta mission ${ }^{11}$. Here we report that neutral Fel and Nil emission lines are ubiquitous in cometary atmospheres, even far from the Sun, as revealed by high-resolution UV-optical spectra of a large sample of comets of various compositions and dynamical origins. The abundances of both species appear to be of the same order of magnitude, contrasting the typical Solar System abundance ratio.
\end{abstract}

The spectra of about 20 different comets were collected since 2002 over a large range of heliocentric distances (0.68 to 3.25 astronomical units) with the UVES spectrograph mounted on the 8-m UT2 telescope of the ESO Very Large Telescope (Extended Data, Table 1). The use of a dichroic beam splitter and a narrow 0.4 " slit provided a resolving power $(\lambda / \Delta \lambda)$ of about 80000 over the wavelength range $304-1040 \mathrm{~nm}$. The slit length of $\sim 10$ "typically covers $\sim 7500 \mathrm{~km}$ of the coma at a distance of 1.0 au and except for a few cases the slit was centered on the comet nucleus. A uniform procedure ${ }^{12}$ has been used for the acquisition and the reduction of all the data.

Close examination of these spectra revealed the omnipresence of emission lines of neutral atoms of iron (Fel) and nickel (Nil) in comets as far as 3.25 au from the Sun with up to about 40 Fel lines and 25 Nil lines for some of them (Extended data, Fig. 1). These lines are weak and located in the blue part of the spectrum $(<450 \mathrm{~nm}$ ) where there are plenty of bright molecular emissions making blends unavoidable, explaining in part why they were missed until now. We searched for the lines of the other metals that were identified in comet Ikeya-Seki ${ }^{7,8}$, in particular neutral chromium, the most abundant after nickel, but we did not find any of them. Nil lines were recently identified in the 
interstellar comet 2l/Borisov (Guzig \& Drahus, this issue).

The metallic lines, contrary to the molecular lines, have only a short spatial extension as it was already noted for Ikeya-Seki ${ }^{7}$, despite very different conditions of temperature and irradiation. The few spectra not centered on the nucleus do not show these lines, or only faintly. In the spectra obtained during the close encounter of comet 103P/Hartley 2 with the Earth (Figure 1), these lines show a radiance inversely proportional to the projected distance to the nucleus $p$. Such a profile corresponds to an ejection from the surface of the nucleus or a short-lived parent ${ }^{5}$ and a constant expansion velocity, resulting in a $p^{-2}$ density distribution. The spectra taken at various position angles indicate that the metals distribution in the inner coma is nearly isotropic, which suggests collisional dragging and an initial velocity high enough to hide radiative pressure effects.

Once freed in a collision-less environment, the atoms are bathed into the solar radiation and would conceivably tend toward an excitation temperature of the order of the color temperature of the Sun, about $5800 \mathrm{~K}$. In order to analyze their emission spectra, we first considered a simple 3-level atomic model as done previously for the analysis of Ikeya-Seki ${ }^{4,13}$. We then built a more realistic multilevel atomic model taking into account the high-resolution structure of the solar spectrum (Supplementary Information). This allowed us to compute the production rates of Nil and Fel for each comet. In the case of an isotropic expansion at a constant velocity $v$, the column densities $C(p)$ and the production rates $Q$ are linked by the relation $C(p)=Q(4 v \Delta p)^{-1}$, where $\Delta$ is the geocentric distance. The observed average density over the slit area $A$ is $N=Q(4 v \Delta A)^{-1} \int_{A} p^{-1}$ da. Due to the atmospheric blurring, we used instead the convolution of the $1 / p$ profile with a $1 "$

Gaussian. We adopted the commonly assumed value of $v=0.85 r^{-1 / 2} \mathrm{~km} / \mathrm{s}$ for the expansion velocity ${ }^{14}$. Extended Data Table 2 gives for each spectrum the Fel and Nil column densities and the corresponding production rates. The quantities found are very small. For the Jupiter family comet $103 \mathrm{P}$ they correspond to only $\sim 1 \mathrm{~g}$ of iron ejected every second, compared to $\sim 100 \mathrm{~kg}$ of water, making these elements minor constituents of the coma.

To compare these abundances to the other usual species observed in comets, we derived from our spectra the production rates of the radicals $\mathrm{OH}$ (a daughter product of water), $\mathrm{CN}$ and $\mathrm{CO}_{2}{ }^{+}$ (Extended DataTable 3), and collected $\mathrm{CO}$ and $\mathrm{H}_{2} \mathrm{O}$ IR and submillimeter measurements from the literature. Figure 2 of Extended Data shows that the abundances of iron and nickel atoms are well correlated with the other species and the comets activity level. Of particular interest is the high metallic abundance in the distant and chemically peculiar comet C/2016 R2 relative to its other elements, except $\mathrm{CO}$ and $\mathrm{CO}_{2}{ }^{+}$. This suggests a possible link between $\mathrm{Fe}, \mathrm{Ni}$ and the carbon oxides. This water-poor comet had a high activity driven by a large $\mathrm{CO}$ production rate of about $10^{29}$ molecules/s at $\sim 3 \mathrm{au}^{15,16}$.

The average Nil/Fel abundance ratio is about unity $(\log (\mathrm{Ni} / \mathrm{Fe})=-0.06 \pm 0.31)$, i.e., an order of magnitude higher than the solar value ${ }^{17}(-1.25 \pm 0.04)$ or the ratio measured in comet lkeya-Seki $(-1.11 \pm 0.09)$ and it does not depend on the heliocentric distance or the comet origin. In our sample, comet $103 \mathrm{P}$ has the lowest value $(-0.64 \pm 0.07)$, and the carbon-chain depleted comet $73 \mathrm{P}$ the

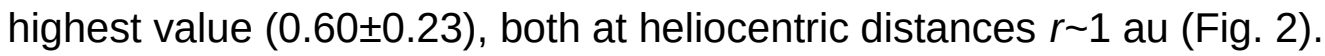


The comet black-body equilibrium temperature of its surface is expected to be around $T \sim 280 r^{-1 / 2}$ $\mathrm{K}$ with $r$ in au, that is $-340 \mathrm{~K}$ for the comet observed at the closest distance to the Sun (0.68 au) and $\sim 150 \mathrm{~K}$ for the most distant one (3.25 au). These temperatures are much lower than those needed to vaporize refractory dust grains as well as iron and nickel in metallic form or in sulfides ${ }^{10}$. We thus explore several possibilities to explain how Fe and $\mathrm{Ni}$ atoms are released at such low temperatures and why the $\mathrm{Ni} / \mathrm{Fe}$ ratio is enhanced.

The $\beta$-parameter characterizing the ratio between the radiation pressure and the gravity, which is about 6 for iron ${ }^{10}$, is too small to alter substantially the velocity field in the vicinity of the nucleus and decrease the column density of iron relative to nickel. Moreover, comet Ikeya-Seki which should show the largest effects, displays a normal (solar) abundance ratio. The high Ni/Fe ratio observed must then be representative of the sublimating material or the sublimation process.

Iron in meteorites is known to be distributed between silicates, sulfides and metallic iron, silicates and metallic iron requiring higher temperatures $(\sim 1200 \mathrm{~K})$ to sublimate than sulfides $(\sim 600 \mathrm{~K})$, while nickel is only found in sulfides and the metal phase ${ }^{18,19}$. We may thus expect a higher Ni/Fe ratio if sublimation occurs at temperatures lower than $1000 \mathrm{~K}$. This is supported by the fact that FeNi alloys and sulfides formed in the low temperature range are Ni-rich, such as kamacite and pentlandite $^{20}$. Although some comets may actually be Ni-rich, partial sublimation of such species could explain the high Ni/Fe ratios we measure in comets far enough from the Sun. Fe and $\mathrm{Ni}$ as well as Ni-rich sulfides like pentlandite have been found in cometary material and interplanetary dust particles (IDPs), often in the form of nanometer-sized particles ${ }^{21-23}$. The number of $\mathrm{Fe}$ and $\mathrm{Ni}$ atoms being the same in such compounds, it would offer an explanation for the relative abundance close to one, in average, but not the large over- or under-abundance of Nil, observed in, e.g., comets Garradd and 103P, or in the carbon-chain depleted comets 21P and 73P. This interpretation requires temperatures above approximately $600 \mathrm{~K}$, still higher than expected at heliocentric distances larger than 0.4 au. However, small grains can be heated at temperatures higher than blackbody equilibrium temperatures, e.g. superheating of submicrometer-sized fluffy aggregates ${ }^{24}$. Even higher temperatures can be reached for smaller grains, like metallic nanoparticles ${ }^{25}$. Collisions of high-velocity nanoparticles with cometary dust grains could break the matrix in which Fe and $\mathrm{Ni}$ are embedded and produce impact vapor with a temperature of the order of $1000 \mathrm{~K}^{26}$. Several mechanisms can thus potentially provide the necessary heating, especially if a significant amount of iron and nickel is in the form of nanoparticles. The refractory elements $\mathrm{Na}, \mathrm{K}, \mathrm{Si}$, and Ca have been found by the Rosetta spacecraft in the gaseous coma of 67P at large distance from the Sun (3 au) and attributed to ion-induced sputtering of the nucleus surface material by the solar wind, but Fe and Ni have not been reported ${ }^{27}$. We do not observe the light refractory elements in our spectra, and nucleus sputtering would not be active for comets closer to the sun due to the much denser coma and would not produce the correlations between $\mathrm{Fe}, \mathrm{Ni}$ and the other volatile species that we observed (Extended data, Fig. 2).

Organometallic complexes such as $[\mathrm{Fe}(\mathrm{PAH})]^{+}$, carbonyls like $\mathrm{Fe}(\mathrm{CO})_{5}$ and $\mathrm{Ni}(\mathrm{CO})_{4}$, and even iron pseudocarbynes, have been proposed as possible constituents of cometary or interstellar material $^{28-31}$. The strong correlation of the production rates of iron, nickel and carbon oxides for all the comets of our sample led us to evaluate the possibility of the carbonyl hypothesis. We 
estimated the sublimation temperatures and sublimation rates of both $\mathrm{Fe}$ and $\mathrm{Ni}$ carbonyls (Extended Data Fig. 3). These temperatures are only slightly higher than that of $\mathrm{CO}_{2}$ and indicate that, if present in comets, these carbonyls can sublimate at low temperatures and at large distances from the Sun, contrary to silicates and sulfides. This could explain why carbonyls have not been found in IDPs while they have been recently identified in the Lewis Cliff 85311 meteorite ${ }^{32}$. Furthermore the higher rate of sublimation of $\mathrm{Ni}(\mathrm{CO})_{4}$ compared to $\mathrm{Fe}(\mathrm{CO})_{5}$ (Extended Data Fig. 3), about a factor 10 at temperatures around $300 \mathrm{~K}$, typical of the diurnal temperature of the nucleus ${ }^{33}$, might provide a simple explanation to the Ni/Fe overabundance, although this scenario depends on the efficiency of the photo-dissociation of the carbonyls. Interestingly, similar computations for $\mathrm{Cr}$, the next most abundant metal in the Sun after $\mathrm{Ni}$, show that the sublimation rate of $\mathrm{Cr}(\mathrm{CO})_{6}$ is lower by a factor of $\sim 100$ with respect to $\mathrm{Fe}(\mathrm{CO})_{5}$, which means that $\mathrm{Crl}$ would be a factor $\sim 10000$ less abundant than Fel, explaining the non-detection of the $\mathrm{Crl}$ lines. A detailed photo-chemical model analysis, beyond the scope of this paper, would be needed to verify if this scenario can actually reproduce the measured abundances, but the discovery of iron and nickel free atoms in comets indicates that important constituents of the nucleus or processes in the coma are still missing, possibly bringing new important constraints on comets composition and the Solar System formation.

\section{References}

1. Zolensky, M. E. et al. Mineralogy and Petrology of Comet 81P/Wild 2 Nucleus Samples. Science 314, 1735 (2006).

2. Bockelée-Morvan, D. \& Biver, N. The composition of cometary ices. Philos. Trans. R. Soc. Lond. Ser. A 375, 20160252 (2017).

3. Copeland, R. \& Lohse, J. G. Spectroscopic observations of comets III and IV, 1881, comet I, 1882, and the Great Comet of 1882. Copernic. Int. J. Astron. 2, 225-244 (1882).

4. Arpigny, C. Relative abundances of the heavy elements in comet Ikeya-Seki /1965 VIII/. in Liege International Astrophysical Colloquia (eds. Boury, A., Grevesse, N. \& Remy-Battiau, L.) vol. 22 189-197 (1979).

5. Combi, M. R., DiSanti, M. A. \& Fink, U. The Spatial Distribution of Gaseous Atomic Sodium in the Comae of Comets: Evidence for Direct Nucleus and Extended Plasma Sources. Icarus 130, 336354 (1997).

6. Dufay, J., Swings, P. \& Fehrenbach, Ch. Spectrographic Observations of Comet Ikeya-Seki (1965f). Astrophys. J. 142, 1698 (1965). 
7. Preston, G. W. The spectrum of Ikeya-Seki (1965f). Astrophys. J. 147, 718-742 (1967).

8. Slaughter, C. D. The Emission Spectrum of Comet Ikeya-Seki 1965-f at Perihelion Passage. Astron. J. 74, 929 (1969).

9. Thackeray, A. D., Feast, M. W. \& Warner, B. Daytime Spectra of Comet Ikeya-Seki Near Perihelion. Astrophys. J. 143, 276 (1966).

10. Fulle, M. et al. Discovery of the Atomic Iron Tail of Comet MCNaught Using the Heliospheric Imager on STEREO. Astrophys. J. Lett. 661, L93-L96 (2007).

11. Kathrin Altwegg and the ROSINA Team. Chemical highlights from the Rosetta mission. Astrochemistry VII - Through the Cosmos from Galaxies to Planets, Proceedings IAU Symposium No. 332 (2017).

12. Arpigny, C. et al. Anomalous Nitrogen Isotope Ratio in Comets. Science 301, 1522-1525 (2003).

13. Arpigny, C. On the nature of comets. In Proceedings of the Robert A. Welch Foundation Conferences on Chemical Research XXI, Cosmochemistry (ed. Mulligan, W. O.) 9 (1978).

14. Cochran, A. L. \& Schleicher, D. G. Observational Constraints on the Lifetime of Cometary $\mathrm{H}_{2} \mathrm{O}$. Icarus 105, 235-253 (1993).

15. Biver, N. et al. Long-term monitoring of the outgassing and composition of comet 67P/Churyumov-Gerasimenko with the Rosetta/MIRO instrument. Astron. Astrophys. 630, A19 (2019).

16. Womack, M., Sarid, G. \& Wierzchos, K. CO in Distantly Active Comets. Publ. Astron. Soc. Pac. 129, 031001 (2017).

17. Lodders, K. Solar Elemental Abundances. https://doi.org/10.1093/acrefore/9780190647926.013.145 (2020).

18. Larimer, J. W. \& Anders, E. Chemical fractionations in meteorites - III. Major element fractionations in chondrites. Geochim. Cosmochim. Acta 34, 367-387 (1970).

19. Grossman, L. \& Larimer, J. W. Early chemical history of the solar system. Rev. Geophys. Space Phys. 12, 71-101 (1974).

20. Lewis, J. S. Physics and chemistry of the solar system. 2nd Edition. (2004). 
21. Berger, E. L., Zega, T. J., Keller, L. P. \& Lauretta, D. S. Evidence for aqueous activity on comet 81P/Wild 2 from sulfide mineral assemblages in Stardust samples and $\mathrm{Cl}$ chondrites. Geo. Cosmo. Acta 75, 3501-3513 (2011).

22. Bradley, J. P. Chemically Anomalous, Preaccretionally Irradiated Grains in Interplanetary Dust From Comets. Science 265, 925-929 (1994).

23. Ishii, H. A. et al. Comparison of Comet 81P/Wild 2 Dust with Interplanetary Dust from Comets. Science 319, 447 (2008).

24. Bockelee-Morvan, D. et al. Comet 67P outbursts and quiescent coma at 1.3 au from the Sun: dust properties from Rosetta/VIRTIS-H observations. Mon. Not. R. Astron. Soc. 469, S443S458 (2017).

25. Hensley, B. S. \& Draine, B. T. Thermodynamics and Charging of Interstellar Iron Nanoparticles. Astrophys. J. 834, 134 (2017).

26. Ip, W.-H. \& Jorda, L. Can the Sodium Tail of Comet Hale-Bopp Have a Dust-Impact Origin? Astrophys. J. Lett. 496, L47-L49 (1998).

27. Wurz, P., et al. Solar wind sputtering of dust on the surface of 67P/Churyumov-Gerasimenko. Astron. Astrophys. 583, p.A22 (2015)

28. Bloch, M. R. \& Wirth, H. L. Abiotic organic synthesis in space. Naturwissenschaften $67,562-$ 564 (1980).

29. Klotz, A. et al. Possible contribution of organometallic species in the solar system ices. Reactivity and spectroscopy. Planet. Space Sci. 44, 957-965 (1996).

30. Huebner, W. F. Dust from cometary nuclei. Astron. Astrophys. 5, 286-297 (1970).

31. Tarakeshwar, P., Buseck, P. R. \& Timmes, F. X. On the Structure, Magnetic Properties, and Infrared Spectra of Iron Pseudocarbynes in the Interstellar Medium. Astrophys. J. 879, 2 (2019).

32. Smith, K. E., House, C. H., Arevalo, R. D., Dworkin, J. P. \& Callahan, M. P. Organometallic compounds as carriers of extraterrestrial cyanide in primitive meteorites. Nat. Commun. 10, 2777 (2019).

33. Prialnik, D., Benkhoff, J. \& Podolak, M. Modeling the structure and activity of comet nuclei. in Comets II (eds. Festou, M. C., Keller, H. U. \& Weaver, H. A.) 359 (2004). 
34. Levison, H. F. Comet Taxonomy. in Completing the Inventory of the Solar System (eds. Rettig, T. \& Hahn, J. M.) vol. 107 173-191 (1996).

35. A'Hearn, M. F., Schleicher, D. G., Millis, R. L., Feldman, P. D. \& Thompson, D. T. Comet Bowell 1980b. Astron. J. 89, 579-591 (1984).

36. Schleicher, D. G. The Fluorescence Efficiencies of the CN Violet Bands in Comets. Astron. J. 140, 973-984 (2010).

37. Schleicher, D. G. \& A'Hearn, M. F. The Fluorescence of Cometary OH. Astrophys. J. 331, 1058 (1988).

38. Bhardwaj, A. \& Raghuram, S. A Coupled Chemistry-emission Model for Atomic Oxygen Green and Red-doublet Emissions in the Comet C/1996 B2 Hyakutake. Astrophys. J. 748, 13 (2012).

39. Raghuram, S. et al. A physico-chemical model to study the ion densitydistribution in the inner coma of comet C/2016 R2(Pan-STARRS). ArXiv E-Prints arXiv:2012.04611 (2020).

40. Weaver, H., Feldman, P., A'Hearn, M., Dello Russo, N. \& Stern, A. Comet 103P/Hartley. Iau circ 9183, 1 (2010), https://ui.adsabs.harvard.edu/abs/2010IAUC.9183...1W.

41. Roth, N. X. et al. Probing the Evolutionary History of Comets: An Investigation of the Hypervolatiles $\mathrm{CO}, \mathrm{CH}_{4}$, and $\mathrm{C}_{2} \mathrm{H}_{6}$ in the Jupiter-family Comet 21P/Giacobini-Zinner. Astron. J. 159, 42 (2020).

42. DiSanti, M. A. et al. Depleted Carbon Monoxide in Fragment C of the Jupiter-Family Comet 73P/Schwassmann-Wachmann 3. Astrophys. J. Lett. 661, L101-L104 (2007).

43. Böhnhardt, H. et al. The Unusual Volatile Composition of the Halley-Type Comet 8P/Tuttle: Addressing the Existence of an Inner Oort Cloud. Astrophys. J. Lett. 683, L71 (2008).

44. Lupu, R. E., Feldman, P. D., Weaver, H. A. \& Tozzi, G.-P. The Fourth Positive System of Carbon Monoxide in the Hubble Space Telescope Spectra of Comets. Astrophys. J. 670, 14731484 (2007).

45. DiSanti, M. A. et al. Detection of Formaldehyde Emission in Comet C/2002 T7 (LINEAR) at Infrared Wavelengths: Line-by-Line Validation of Modeled Fluorescent Intensities. Astrophys. J. 
650, 470-483 (2006).

46. Paganini, L. et al. Observations of Comet C/2009 P1 (Garradd) at 2.4 and 2.0 AU before Perihelion. in Asteroids, Comets, Meteors 2012 vol. 16676331 (2012).

47. Paganini, L. et al. The Unexpectedly Bright Comet C/2012 F6 (Lemmon) Unveiled at Nearinfrared Wavelengths. Astron. J. 147, 15 (2014).

48. Biver, N. et al. The extraordinary composition of the blue comet C/2016 R2 (PanSTARRS). Astron. Astrophys. 619, A127 (2018).

49. Wierzchos, K. \& Womack, M. C/2016 R2 (PANSTARRS): A Comet Rich in CO and Depleted in HCN. Astron. J. 156, 34 (2018).

50. Faggi, S., Mumma, M. J., Villanueva, G. L., Paganini, L. \& Lippi, M. Quantifying the Evolution of Molecular Production Rates of Comet 21P/Giacobini-Zinner with iSHELL/NASA-IRTF. Astron. J. 158, 254 (2019).

51. de Val-Borro, M. et al. A survey of volatile species in Oort cloud comets C/2001 Q4 (NEAT) and C/2002 T7 (LINEAR) at millimeter wavelengths. Astron. Astrophys. 559, A48 (2013).

52. Yamamoto, T., Nakagawa, N. \& Fukui, Y. The chemical composition and thermal history of the ice of a cometary nucleus. Astron. Astrophys. 122, 171-176 (1983).

53. Yamamoto, T. Formation environment of cometary nuclei in the primordial solar nebula. Astron. Astrophys. 142, 31-36 (1985).

54. Gilbert, A. G. \& Sulzmann, K. G. P. The Vapor Pressure of Iron Pentacarbonyl. J. Electrochem. Soc. 121, 832 (1974).

55. Stull, D. R. Vapor Pressure of Pure Substances. Organic and Inorganic Compounds. Ind Eng Chem 39, 517 (1947).

56. Delsemme, A. H. Chemical composition of cometary nuclei. in IAU Colloq. 61: Comet Discoveries, Statistics, and Observational Selection (ed. Wilkening, L. L.) 85-130 (1982). 


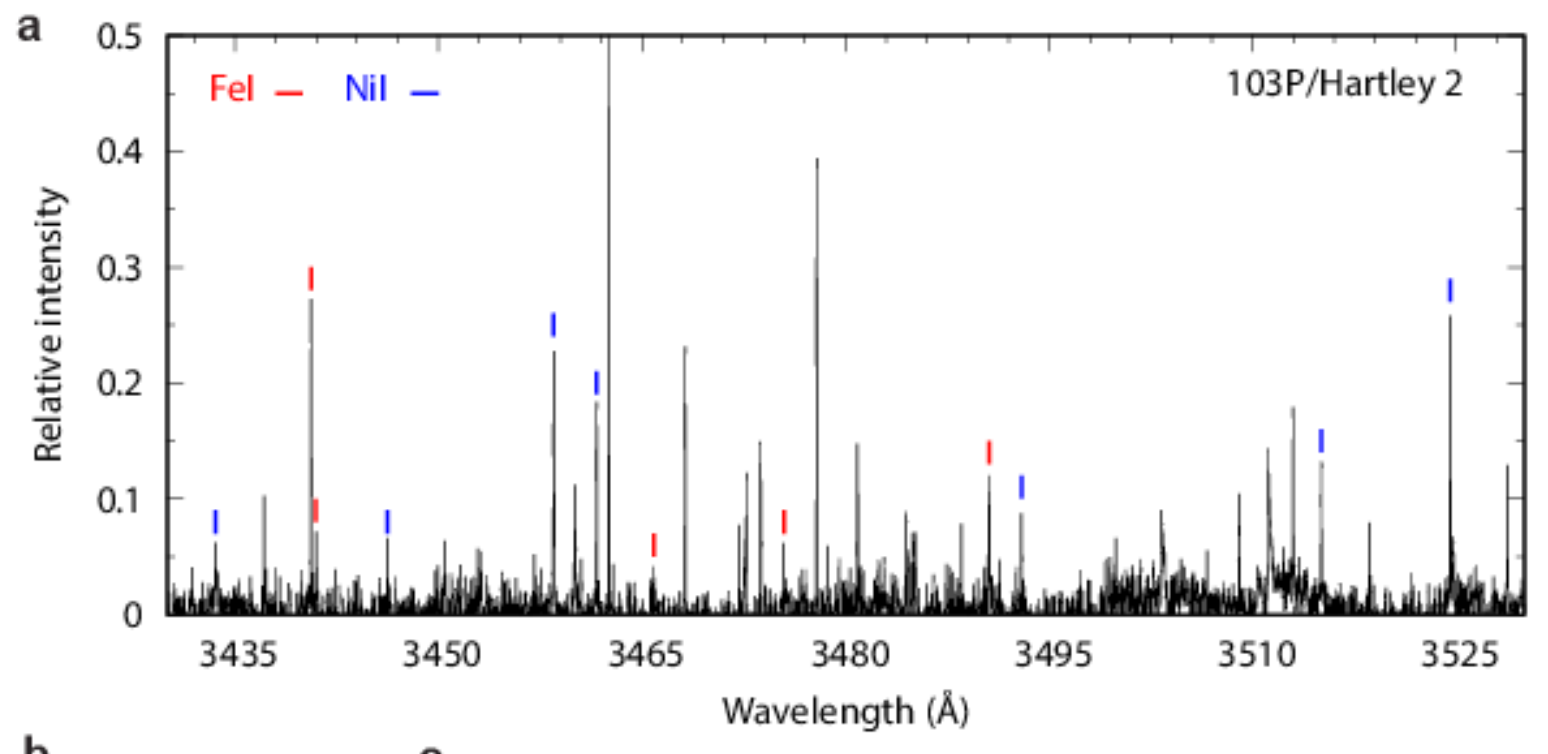

b

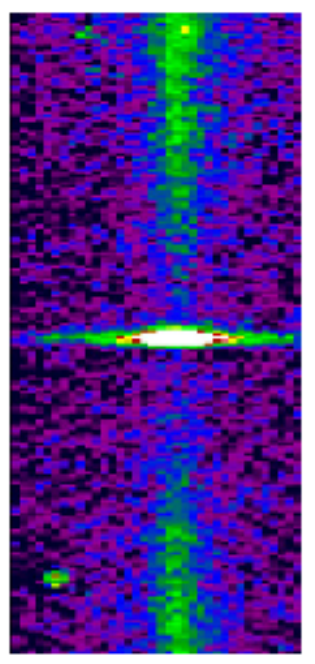

C

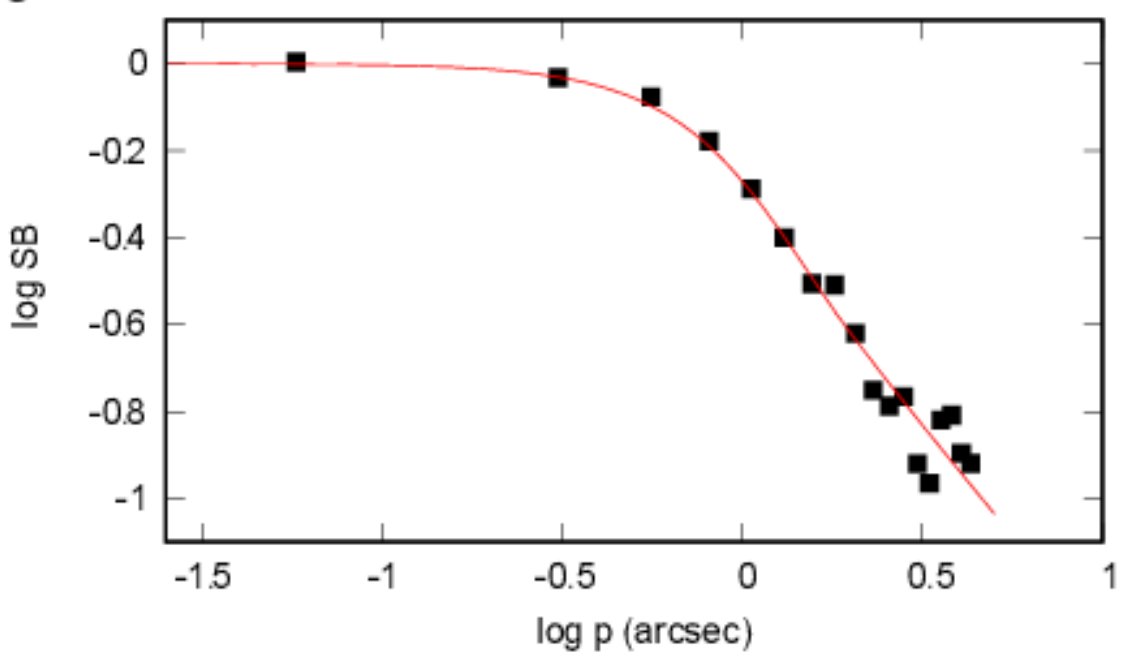

Figure 1: Example of Fel and Nil lines in comet 103P/Hartley 2. a, Selected spectral region showing many Fel and Nil lines in the spectrum of the Jupiter family comet 103P/Hartley 2 obtained at the ESO VLT in April 2004 at 0.17 au from Earth. b, 2-dimensional spectrum of the Fel $3719 \AA$ line. Wavelengths are along the vertical axis and cover a range of $3 \AA$. The spatial dimension (horizontal axis) extends over the entire length of the 10 " slit (1230 km at the distance of the comet). The vertical trace represents the solar spectrum reflected by the dust and shows the deep photospheric absorption of the Fel 3719 line. c, The spatial profile of the same line plotted as a function of the projected nucleocentric distance $p$ agrees well with a 1/p distribution of the surface brightness (SB) and a 1.35" blurring corresponding to the seeing and the tracking imperfections. The Nil lines display the same kind of profile. 


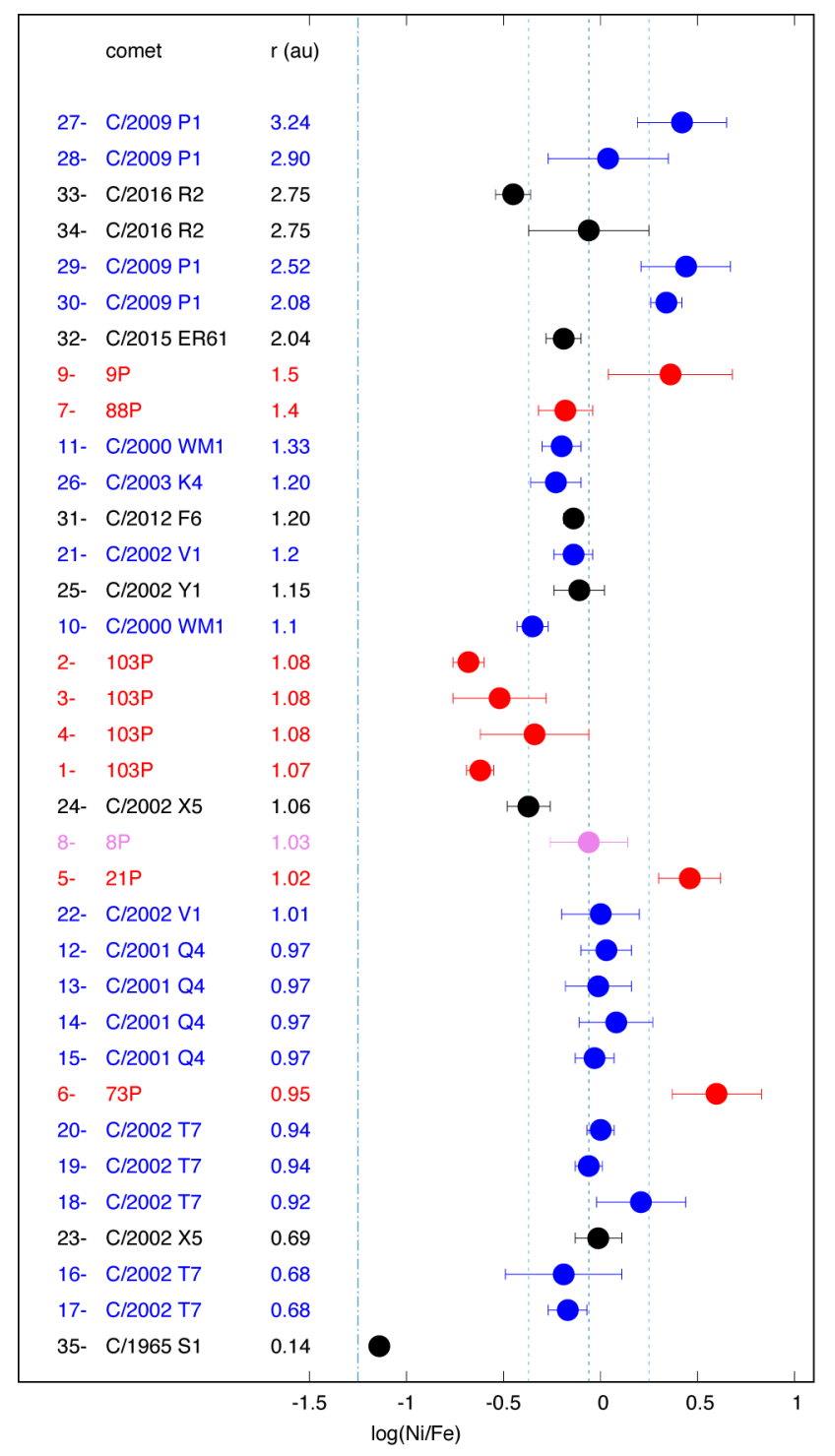

Figure 2: Ni/Fe abundance ratios from the multilevel model versus heliocentric distance. The best spectra have been selected and the great comet C/1965 S1 (Ikeya-Seki) has been added as number 35 . The vertical dashed-dotted line indicates the solar value and the dotted lines represent the sample average and standard deviation. Different colors are used according to the dynamical class $^{34}$ : Jupiter family comets in red and Halley family comets in pink correspond to ecliptic comets with short periods ( $<200$ years), external comets in blue have a semi-major axis $a<10000$ au and new comets in black come directly come from the Oort cloud ( $a>10000 \mathrm{au}$ ). 


\begin{tabular}{|c|c|c|c|c|c|c|c|c|c|c|c|c|}
\hline & Comet & Type & Dates & $\begin{array}{c}\lambda \text { range } \\
\AA\end{array}$ & $N$ & $\begin{array}{r}r \\
\text { au }\end{array}$ & $\begin{array}{r}\dot{r} \\
\mathrm{~km} / \mathrm{s}\end{array}$ & $\begin{array}{r}\Delta \\
\text { au }\end{array}$ & $\begin{array}{r}\dot{\Delta} \\
\mathrm{km} / \mathrm{s}\end{array}$ & $\begin{array}{l}d \\
\prime \prime\end{array}$ & $\begin{array}{l}w \\
\prime \prime\end{array}$ & $\begin{array}{l}h \\
\prime \prime\end{array}$ \\
\hline 2 & & JFC & 2010-11-11 & $3105-3910$ & 1 & 1.08 & 4.4 & 0.19 & 8.2 & 10 & 0.44 & 9.5 \\
\hline 4 & & JFC & 2010-11-11 & $3105-3910$ & 1 & 1.08 & 4.4 & 0.19 & 8.2 & 30 & 0.44 & 9.5 \\
\hline 5 & 21P/Giacobini-Zinner & JFC & 2018-09-18 & 3104-3879 & 1 & 1.02 & 2.9 & 0.40 & 4.2 & 0 & 0.44 & 9.5 \\
\hline 6 & 73P/Schwassmann-Wachmann 3 & JFC & 2006-05-27 & 3104-3911 & 1 & 0.95 & -4.2 & 0.15 & 12.3 & 0 & 0.60 & 9.5 \\
\hline 7 & 88P/Howell & JFC & 2004-04-18:-05-24 & $3286-4513$ & 11 & 1.40 & 3.0 & 1.65 & -3.4 & 0 & 0.44 & 7.5 \\
\hline 10 & C/2000 WM 1 (LINEAR) & NEW & 2002-03-07:-03-08 & $3286-4513$ & 4 & 1.10 & 28.3 & 1.24 & 0.2 & 10 & 0.45 & 7.5 \\
\hline 11 & & NEW & 2002-03-22:-03-23 & $3286-4513$ & 4 & 1.33 & 27.9 & 1.24 & -0.2 & 2 & 0.45 & 7.5 \\
\hline 12 & C/2001 Q4 (NEAT) & NEW & 2004-05-05 & $3286-4513$ & 1 & 0.98 & -5.4 & 0.32 & -4.1 & 3 & 0.44 & 9.5 \\
\hline 13 & & NEW & 2004-05-06 & $3286-4513$ & 1 & 0.98 & -5.4 & 0.32 & -3.3 & 13 & 0.44 & 7.5 \\
\hline 14 & & NEW & 2004-05-06 & $3286-4513$ & 1 & 0.97 & -4.9 & 0.32 & 1.9 & 200 & 0.44 & 7.5 \\
\hline 15 & & NEW & 2004-05-07 & $3286-4513$ & 1 & 0.97 & -4.8 & 0.32 & 2.2 & 0 & 0.44 & 9.5 \\
\hline 20 & & NEW & 2004-05-27 & 3104-3878 & 1 & 0.94 & 25.6 & 0.42 & 55.2 & 0 & 0.40 & 9.5 \\
\hline 21 & $\mathrm{C} / 2002 \mathrm{Vl}$ (NEAT) & NEW & 2003-01-08:-01-10 & $3286-4513$ & 4 & 1.20 & -37.0 & 0.84 & 8.0 & 0 & 0.45 & 7.5 \\
\hline 22 & & NEW & 2003-03-21 & $3286-4513$ & 1 & 1.01 & 39.8 & 1.63 & 42.0 & 0 & 0.45 & 7.5 \\
\hline 23 & C/2002 X5 (Kudo-Fujikawa) & EXT & 2003-02-19 & $3286-4513$ & 1 & 0.70 & 43.0 & 0.86 & -5.0 & 0 & 0.45 & 7.5 \\
\hline 24 & & EXT & 2003-03-07 & $3286-4513$ & 3 & 1.06 & 37.0 & 0.99 & 29.4 & 3 & 0.45 & 7.5 \\
\hline 25 & C/2002 Y1 (Juels-Holvorcem) & EXT & 2003-05-29:-05-30 & $3286-4513$ & 4 & 1.15 & 24.1 & 1.55 & -7.2 & 2 & 0.40 & 7.5 \\
\hline 26 & C/2003 K4 (LINEAR) & NEW & 2004-05-04 & 3104-3879 & 1 & 1.20 & 14.8 & 1.51 & -28.2 & 0 & 0.44 & 9.5 \\
\hline 27 & C/2009 Pl (Garradd) & NEW & 2011-05-11 & 3105-3911 & 1 & 3.25 & -16.9 & 3.50 & -44.7 & 0 & 0.44 & 9.5 \\
\hline 28 & & NEW & 2011-06-15 & 3105-3911 & 1 & 2.90 & -16.9 & 2.57 & -46.4 & 0 & 0.44 & 9.5 \\
\hline 29 & & NEW & 2011-07-25 & 3105-3885 & 1 & 2.52 & -16.5 & 1.64 & -29.3 & 0 & 0.44 & 9.5 \\
\hline 30 & & NEW & 2011-09-10:-09-12 & $3044-3916$ & 3 & 2.08 & -14.8 & 1.48 & 15.1 & 0 & 0.44 & 9.5 \\
\hline 31 & C/2012 F6 (Lemmon) & EXT & 2013-02-02 & 3104-3911 & 3 & 1.20 & -24.0 & 0.99 & -4.5 & 0 & 0.44 & 9.5 \\
\hline 32 & C/2015 ER61 & EXT & 2017-04-13:-04-15 & $3104-3879$ & 2 & 2.04 & 6.8 & 1.94 & -2.0 & 0 & 0.44 & 9.5 \\
\hline
\end{tabular}

Extended Data Table 1. Comets observing circumstances with UVES at ESO VLT. In several cases, $N$ spectra have been averaged. $r$ and $\Delta$ are the heliocentric and geocentric distances in au and the dotted symbols are the corresponding velocities in $\mathrm{km} / \mathrm{s}$. The offset $d$ from the nucleus, the slit width $w$ and the slit height $h$ are given in arc seconds. Type refers to their dynamical class as defined by ${ }^{34}$ : JFC (Jupiter Family) and HFC (Halley Family) correspond to ecliptic comets with short periods (<200 years), EXT corresponds to external comets with semi-major axis $a<10000$ au and NEW corresponds to external comets which directly come from the Oort cloud ( $a>10000 \mathrm{au}$ ). 


\begin{tabular}{|c|c|c|c|c|c|c|c|c|c|}
\hline \multirow[b]{2}{*}{ ID } & \multirow[b]{2}{*}{ Comet } & \multirow{2}{*}{$\begin{array}{c}n_{\text {lines }} \\
\text { (FeI / Nil) }\end{array}$} & \multicolumn{2}{|c|}{ 3-level model } & \multicolumn{5}{|c|}{ multi-level model } \\
\hline & & & $\mathrm{T}(\mathrm{K})$ & $\log (\mathrm{Ni} / \mathrm{Fe})^{*}$ & $\log (N[\mathrm{Fe}])$ & $\log (N[\mathrm{NiI}])$ & $\log (Q[\mathrm{FeI}])$ & $\log (Q[\mathrm{NiI}])$ & $\log (\mathrm{NiI} / \mathrm{FeI})^{* * *}$ \\
\hline 2 & & $16 / 7$ & $3290 \pm 180$ & $-0.47 \pm 0.08$ & $8.33 \pm 0.05$ & $7.65 \pm 0.06$ & $21.98 \pm 0.05$ & $21.30 \pm 0.06$ & $-0.68 \pm 0.08$ \\
\hline 3 & & $10 / 2$ & $4791 \pm 1278$ & $-0.27 \pm 0.27$ & $8.05 \pm 0.12$ & $7.53 \pm 0.21$ & $22.01 \pm 0.12$ & $21.48 \pm 0.21$ & $-0.52 \pm 0.24$ \\
\hline 5 & $21 \mathrm{P}$ & $8 / 23$ & $3017 \pm 590$ & $0.63 \pm 0.16$ & $8.74 \pm 0.14$ & $9.20 \pm 0.07$ & $21.85 \pm 0.14$ & $22.30 \pm 0.07$ & $0.46 \pm 0.16$ \\
\hline 6 & $73 \mathrm{P}$ & $6 / 15$ & $2785 \pm 1185$ & $0.98 \pm 0.28$ & $8.54 \pm 0.21$ & $9.14 \pm 0.09$ & $21.23 \pm 0.21$ & $21.83 \pm 0.09$ & $0.60 \pm 0.23$ \\
\hline 7 & $88 \mathrm{P}$ & $21 / 13$ & $4212 \pm 782$ & $0.01 \pm 0.18$ & $8.61 \pm 0.09$ & $8.43 \pm 0.11$ & $21.78 \pm 0.09$ & $21.60 \pm 0.11$ & $-0.18 \pm 0.14$ \\
\hline 10 & $\mathrm{C} / 2000 \mathrm{WM} 1$ & $14 / 7$ & $4636 \pm 463$ & $-0.06 \pm 0.14$ & $8.17 \pm 0.06$ & $7.82 \pm 0.06$ & $22.63 \pm 0.06$ & $22.28 \pm$ & 08 \\
\hline 11 & & $13 / 8$ & $4272 \pm 537$ & $0.11 \pm$ & 8.2 & $8 . C$ & $22.12 \pm 0.07$ & 21. & -0 \\
\hline 12 & $\mathrm{C} / 2001 \mathrm{Q} 4$ & $13 / 10$ & $4213 \pm 530$ & $0.27 \pm 0.14$ & $9.70=$ & $9.72 \pm 0.11$ & $23.18 \pm 0.07$ & 23.20 & 0 \\
\hline 13 & & $8 / 8$ & $3519 \pm 589$ & $0.15 \pm 0.19$ & $9.07 \pm 0.12$ & $9.07 \pm 0.12$ & $23.09 \pm 0.12$ & 23.08 & -0 \\
\hline 14 & & $7 / 4$ & $5069 \pm 866$ & $0.33 \pm 0.23$ & $7.76=$ & .18 & $22.96 \pm 0.07$ & 23.0 & 0. \\
\hline 15 & & $17 / 13$ & $3481 \pm 320$ & $0.13 \pm 0.12$ & $9.27 \pm 0.07$ & $9.25 \pm 0.07$ & $22.29 \pm 0.07$ & $22.26 \pm 0.07$ & $-0.03 \pm 0.10$ \\
\hline 16 & $\mathrm{C} / 2002 \mathrm{~T} 7$ & $2 / 2$ & $4000 \pm 1000$ & $0.20 \pm 0.35$ & $9.68 \pm 0.21$ & $9.49 \pm 0.21$ & $23.68 \pm 0.21$ & $23.49 \pm 0.21$ & $-0.19 \pm 0.30$ \\
\hline 21 & $\mathrm{C} / 2002 \mathrm{~V} 1$ & 15 & \pm 419 & $0.08 \pm$ & & 99 & 05 & 09 & -0 . \\
\hline 22 & & 19 & \pm 1776 & $-0.01 \pm$ & & 15 & 13 & 22. & 0 \\
\hline 23 & C/2002 X5 & $19 / 16$ & $4115 \pm 459$ & $0.08 \pm 0.13$ & 8.4 & 8.4 & $21.94 \pm 0.07$ & 21.93 & -0 \\
\hline 24 & & $10 / 12$ & $4734 \pm 713$ & $-0.20 \pm 0.15$ & $8.27 \pm 0.08$ & $7.90 \pm 0.08$ & $22.20 \pm 0.08$ & $21.83 \pm 0.08$ & $-0.37 \pm 0.11$ \\
\hline 25 & $\mathrm{C} / 2002 \mathrm{Y} 1$ & $15 / 11$ & $3339 \pm 335$ & $0.04 \pm 0.16$ & $8.60 \pm 0.05$ & $8.49 \pm 0.12$ & $22.58 \pm 0.05$ & $22.47 \pm 0.12$ & $-0.11 \pm 0.13$ \\
\hline 26 & C/2003 K4 & $3 / 10$ & $4000 \pm 1000$ & $0.24 \pm 0.16$ & $8.46 \pm 0.09$ & $8.23 \pm 0.09$ & $22.10 \pm 0.09$ & $21.87 \pm 0.09$ & $-0.23 \pm 0.13$ \\
\hline 27 & C/2009 P1 & $2 / 13$ & $4000 \pm 1000$ & $0.85 \pm 0.28$ & $8.19 \pm 0.21$ & $8.61 \pm 0.09$ & $21.97 \pm 0.21$ & $22.39 \pm 0.09$ & .23 \\
\hline 28 & & $1 / 7$ & $4000 \pm 1000$ & $0.47 \pm 0.36$ & $8.65 \pm 0.30$ & $8.68 \pm 0.09$ & $22.33 \pm 0.30$ & $22.37 \pm 0.09$ & $=0.31$ \\
\hline 29 & & $2 / 14$ & $4000 \pm 1000$ & $0.69 \pm 0.27$ & $8.62 \pm 0.21$ & $9.05 \pm 0.08$ & $22.13 \pm 0.21$ & $22.57 \pm 0.08$ & $0.44 \pm 0.23$ \\
\hline 30 & & $11 / 21$ & 512 & $0.65 \pm$ & 8.7 & 9.1 & $22.28=$ & 22. & \\
\hline 31 & $\mathrm{C} / 2012 \mathrm{~F} 6$ & $27 / 23$ & $4288 \pm 340$ & $0.13 \pm 0.08$ & $9.21 \pm 0.03$ & $9.07 \pm 0.04$ & $22.66 \pm 0.03$ & $22.52 \pm 0.04$ & $=0.05$ \\
\hline 32 & C/2015 ER61 & $19 / 15$ & $3660 \pm 452$ & $0.07 \pm 0.12$ & $9.42 \pm 0.07$ & $9.24 \pm 0.06$ & $23.05 \pm 0.07$ & $22.87 \pm 0.06$ & -0.19 \\
\hline 33 & C/2016 R2 & $43 / 18$ & $3477 \pm 138$ & $-0.27 \pm 0.09$ & $9.87 \pm 0.04$ & $9.42 \pm 0.08$ & $23.46 \pm 0.04$ & $23.01 \pm 0.08$ & $-0.45 \pm 0.09$ \\
\hline
\end{tabular}

Extended Data Table 2. Ni/Fe abundance ratios from the 3-level and the multilevel models. $T$ is the excitation temperature used for both Fel and Nil, and $n_{\text {lines }}$ the number of Fel and Nil lines considered in the analysis. The column densities $N\left(\right.$ atoms $\left./ \mathrm{cm}^{2}\right)$ and the production rates $Q$ (atoms/s) come from the multilevel model. 


\begin{tabular}{|c|c|c|c|c|c|}
\hline & Comet & $\log \left(Q\left[\mathrm{CO}_{2}^{+}\right]\right)$ & $\log (Q[\mathrm{OH}])$ & $\log (Q[\mathrm{CN}])$ & $\log (A f \rho)$ \\
\hline 1 & $103 \mathrm{P}$ & $25.89 \pm 0.16$ & $27.65 \pm 0.02$ & $24.72 \pm 0.02$ & $1.16 \pm 0.09$ \\
\hline 2 & & $25.90 \pm 0.33$ & $27.74 \pm 0.05$ & $24.77 \pm 0.03$ & $1.65 \pm 0.11$ \\
\hline 3 & & $25.71 \pm 0.31$ & $27.72 \pm 0.07$ & $24.76 \pm 0.04$ & $1.70 \pm 0.21$ \\
\hline 4 & & & $27.76 \pm 0.07$ & $24.83 \pm 0.04$ & $1.72 \pm 0.15$ \\
\hline 5 & $21 \mathrm{P}$ & $26.11 \pm 0.32$ & $27.91 \pm 0.02$ & $25.18 \pm 0.05$ & $2.05 \pm 0.05$ \\
\hline 6 & $73 \mathrm{P}$ & & $27.85 \pm 0.01$ & $25.30 \pm 0.04$ & $1.26 \pm 0.06$ \\
\hline 7 & $88 \mathrm{P}$ & $26.76 \pm 0.12$ & & $25.10 \pm 0.04$ & $2.91 \pm 0.31$ \\
\hline 8 & $8 \mathrm{P}$ & & $27.90 \pm 0.01$ & $25.28 \pm 0.03$ & $2.19 \pm 0.13$ \\
\hline 9 & $9 \mathrm{P}$ & & $27.14 \pm 0.06$ & $24.50 \pm 0.03$ & $2.33 \pm 0.01$ \\
\hline 10 & $\mathrm{C} / 2000 \mathrm{WM} 1$ & $27.04 \pm 0.14$ & & $25.81 \pm 0.03$ & $3.81 \pm 0.06$ \\
\hline 11 & & $26.56 \pm 0.32$ & & $25.47 \pm 0.03$ & $3.31 \pm 0.07$ \\
\hline 12 & $\mathrm{C} / 2001 \mathrm{Q} 4$ & & & $26.01 \pm 0.02$ & $3.36 \pm 0.06$ \\
\hline 13 & & $26.79 \pm 0.24$ & & $26.14 \pm 0.03$ & $3.36 \pm 0.04$ \\
\hline 14 & & & & $26.43 \pm 0.02$ & $3.22 \pm 0.07$ \\
\hline 15 & & $26.93 \pm 0.20$ & & $25.91 \pm 0.02$ & $2.32 \pm 0.05$ \\
\hline 16 & $\mathrm{C} / 2002 \mathrm{~T} 7$ & & & $26.55 \pm 0.02$ & $3.55 \pm 0.04$ \\
\hline 17 & & $27.18 \pm 0.29$ & $29.05 \pm 0.00$ & $26.64 \pm 0.02$ & $3.68 \pm 0.06$ \\
\hline 18 & & & & $25.88 \pm 0.04$ & $2.52 \pm 0.05$ \\
\hline 19 & & $27.09 \pm 0.18$ & $28.69 \pm 0.00$ & $26.06 \pm 0.02$ & $2.74 \pm 0.04$ \\
\hline 20 & & $27.00 \pm 0.24$ & $28.65 \pm 0.01$ & $26.02 \pm 0.02$ & $2.71 \pm 0.04$ \\
\hline 21 & $\mathrm{C} / 2002 \mathrm{~V} 1$ & $26.37 \pm 0.42$ & & $25.34 \pm 0.02$ & $2.52 \pm 0.21$ \\
\hline 22 & & & & $25.84 \pm 0.02$ & $3.80 \pm 0.06$ \\
\hline 23 & $\mathrm{C} / 2002 \times 5$ & $26.63 \pm 0.14$ & & $25.73 \pm 0.04$ & $2.75 \pm 0.06$ \\
\hline 24 & & $26.74 \pm 0.12$ & & $25.38 \pm 0.04$ & $2.88 \pm 0.03$ \\
\hline 25 & $\mathrm{C} / 2002 \mathrm{Y} 1$ & $27.03 \pm 0.18$ & & $25.84 \pm 0.02$ & $3.71 \pm 0.03$ \\
\hline 26 & $\mathrm{C} / 2003 \mathrm{~K} 4$ & & $28.55 \pm 0.01$ & $25.83 \pm 0.02$ & $3.68 \pm 0.07$ \\
\hline 27 & $\mathrm{C} / 2009 \mathrm{P} 1$ & & & $25.37 \pm 0.02$ & $4.26 \pm 0.07$ \\
\hline 28 & & & $27.55 \pm 0.23$ & $25.47 \pm 0.04$ & $4.42 \pm 0.09$ \\
\hline 29 & & & $28.10 \pm 0.15$ & $25.64 \pm 0.06$ & $3.68 \pm 0.08$ \\
\hline 30 & & & $28.37 \pm 0.04$ & $25.85 \pm 0.04$ & $4.05 \pm 0.03$ \\
\hline 31 & $\mathrm{C} / 2012 \mathrm{~F} 6$ & $27.13 \pm 0.39$ & $28.90 \pm 0.01$ & $26.16 \pm 0.09$ & $3.62 \pm 0.11$ \\
\hline 32 & C/2015 ER61 & $27.27 \pm 0.42$ & $29.31 \pm 0.01$ & $26.31 \pm 0.06$ & $4.39 \pm 0.02$ \\
\hline 33 & $\mathrm{C} / 2016 \mathrm{R} 2$ & $28.13 \pm 0.11$ & $27.73 \pm 0.36$ & $24.44 \pm 0.24$ & $3.23 \pm 0.12$ \\
\hline 34 & & & $27.73 \pm 0.36$ & $24.32 \pm 0.07$ & $3.25 \pm 0.12$ \\
\hline
\end{tabular}

Extended Data Table 3. Production rates of molecules and dust. Logarithm of the production rates of the gaseous species $\mathrm{OH}, \mathrm{CN}, \mathrm{CO}_{2}{ }^{+}$(molecules/s), and, as a proxy of the dust production rate, the $A f \rho$ parameter ${ }^{35}$, to which it is proportional for a $1 / p$ brightness profile. Af $\rho$ is derived from the dust continuum intensity around the $\mathrm{CN}$ band and is corrected for the phase effect, the seeing and the slit geometry. The production rates of $\mathrm{OH}, \mathrm{CN}$ and $\mathrm{CO}_{2}{ }^{+}$were derived from a Haser model. For the $\mathrm{OH}$ and the $\mathrm{CN}$ bands, we used, respectively, the data from ${ }^{36,37}$ for the $A_{2} \Sigma^{+}-X_{2} \Pi(0,0)$ band near $3090 \AA$ and the $\mathrm{B}_{2} \Sigma^{+}-\mathrm{X}_{2} \Sigma^{+}(0,0)$ band near $3880 \AA$. For the $\mathrm{CO}_{2}{ }^{+} \mathrm{A}_{2} \Pi-\mathrm{X}_{2} \Pi(0,0)$ band near $3500 \AA$ we assumed a simple Haser profile with one component, $\mathrm{CO}_{2}$ being the parent. The time scale and fluorescence efficiency are taken from ${ }^{38,39}$. The profile distribution was integrated over the area of the slit of the spectrograph and compared to the observed flux of the bands to yield the production rate. 

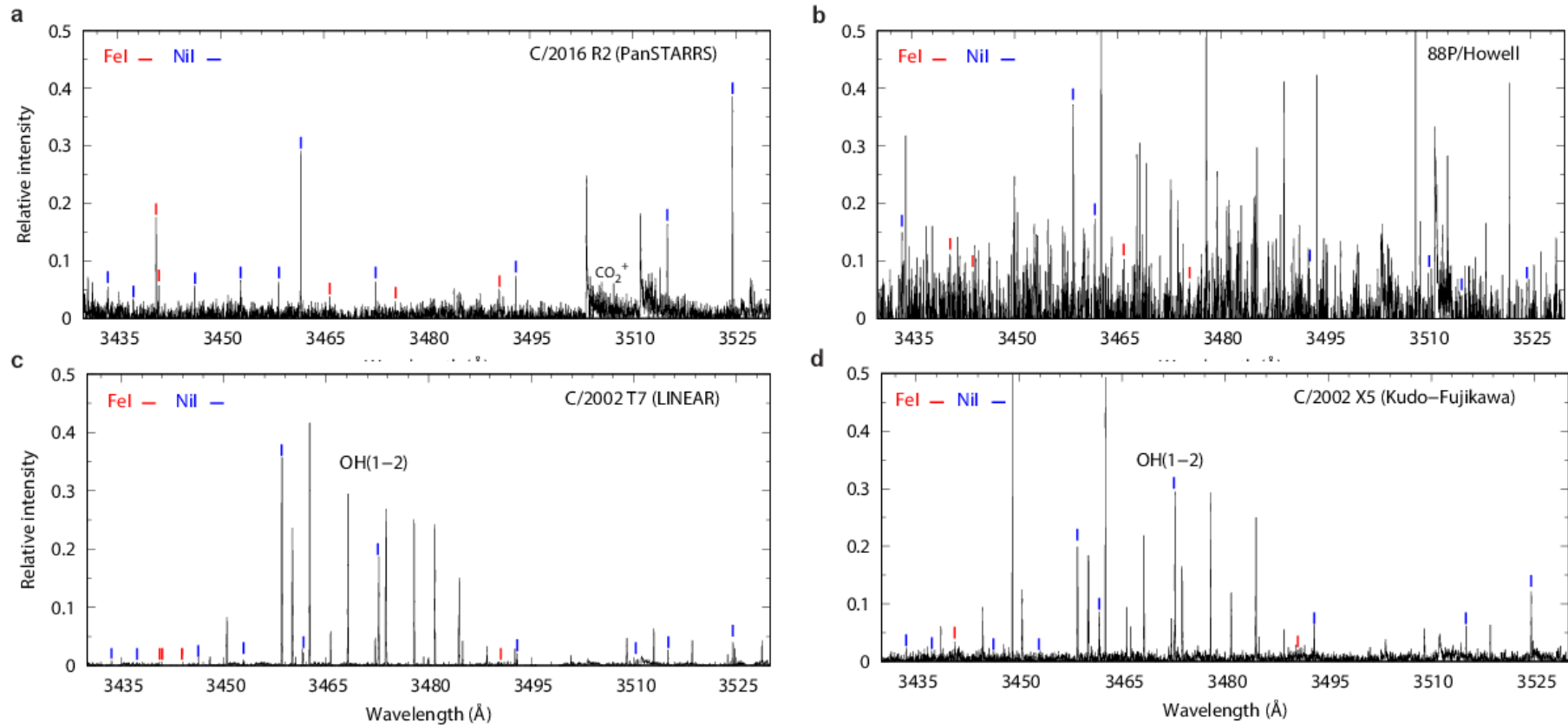

d

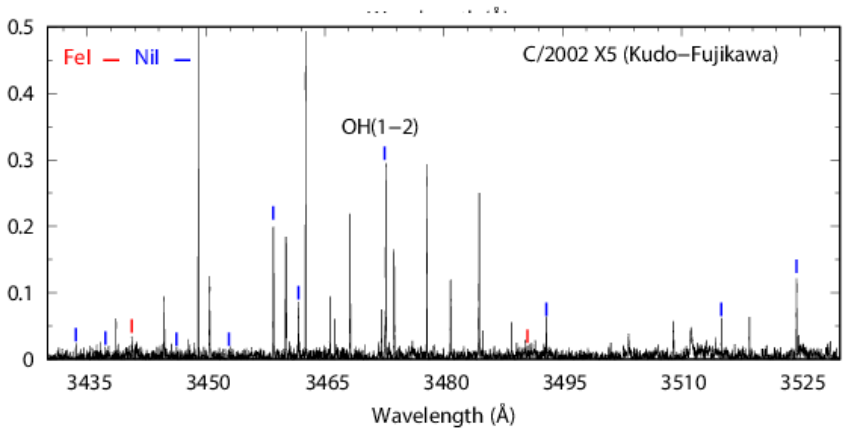

Extended Data Fig. 1. Examples of UVES comet spectra. Comet spectra obtained with the UVES spectrograph at ESO VLT, showing many $\mathrm{Fe} i$ and $\mathrm{Ni} i$ lines in the selected wavelength region (3,425-3,530 $)$ ). a, Spectrum of the water-poor and CO-rich long-period comet C/2016 R2 (PanSTARRS) at 3 au. b, Spectrum of the Jupiter-family comet 88P/Howell at 1.4 au. $\mathbf{c}$, Spectrum of the new comet $\mathrm{C} / 2002 \mathrm{T7}$ (LINEAR) at $0.68 \mathrm{au}$, with lines from the $\mathrm{OH}(1-2)$ band. $\mathbf{d}$, Spectrum of the long-period comet $\mathrm{C} / 2020 \times 5$ (Kudo-Fujikawa). Fe $\mathrm{i}$ and $\mathrm{Ni} \mathrm{i}$ lines are indicated by red and blue marks, respectively. 

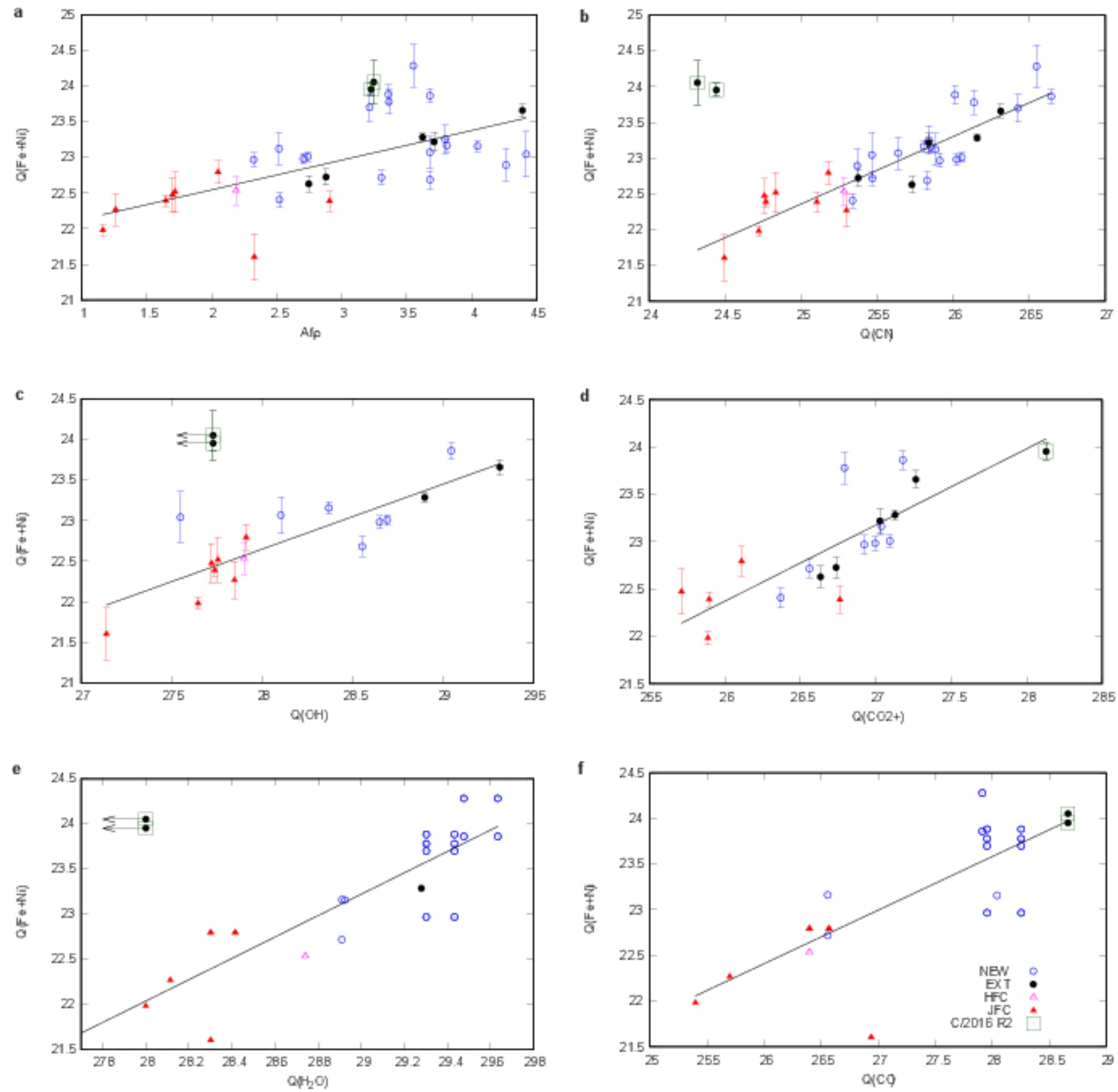

Extended Data Fig. 2. Comparisons of $\mathrm{Fe} \mathrm{I}, \mathrm{Ni} I$ and dust production rates. a-d, The production rates of Fel and Nil are compared to Af $\rho$ which is the product of the reflectivity of the grains, their filling factor and the radius of the coma (used as a proxy to the dust production rate) and to the production rates of $\mathrm{OH}, \mathrm{CN}$ and $\mathrm{CO}_{2}{ }^{+}$as determined from our spectra. e-f, The production rates of Fel and Nil are compared to the production rates of $\mathrm{H}_{2} \mathrm{O}$ and $\mathrm{CO}$ measured by various authors in comets 8P, 9P, 21P, 73P, 103P, C/2000 WM1, C/2001 Q4, C/2002 T7, C/2009 P1, C/2012 F6 and C/2016 R2 at about the same epochs as our observations ${ }^{40-51}$. The various cometary types are color coded according to their dynamical classification (see Extended Data Table 1). The $\mathrm{OH}$ and $\mathrm{H}_{2} \mathrm{O}$ values relative to comet $\mathrm{C} / 2016 \mathrm{R} 2$ are upper limits. The Pearson's correlation coefficients calculated without (and with) the C/2016 R2 data are $\rho_{\mathrm{OH}}=0.844(0.531)$, $\rho_{\text {Afp }}=0.644(0.616), \rho_{\mathrm{CN}}=0.892(0.518), \rho_{\mathrm{CO} 2}{ }^{+}=0.755(0.804), \rho_{\mathrm{H} 2 \mathrm{O}}=0.849(0.627)$ and $\rho_{\mathrm{CO}}=0.752$ 
(0.770).
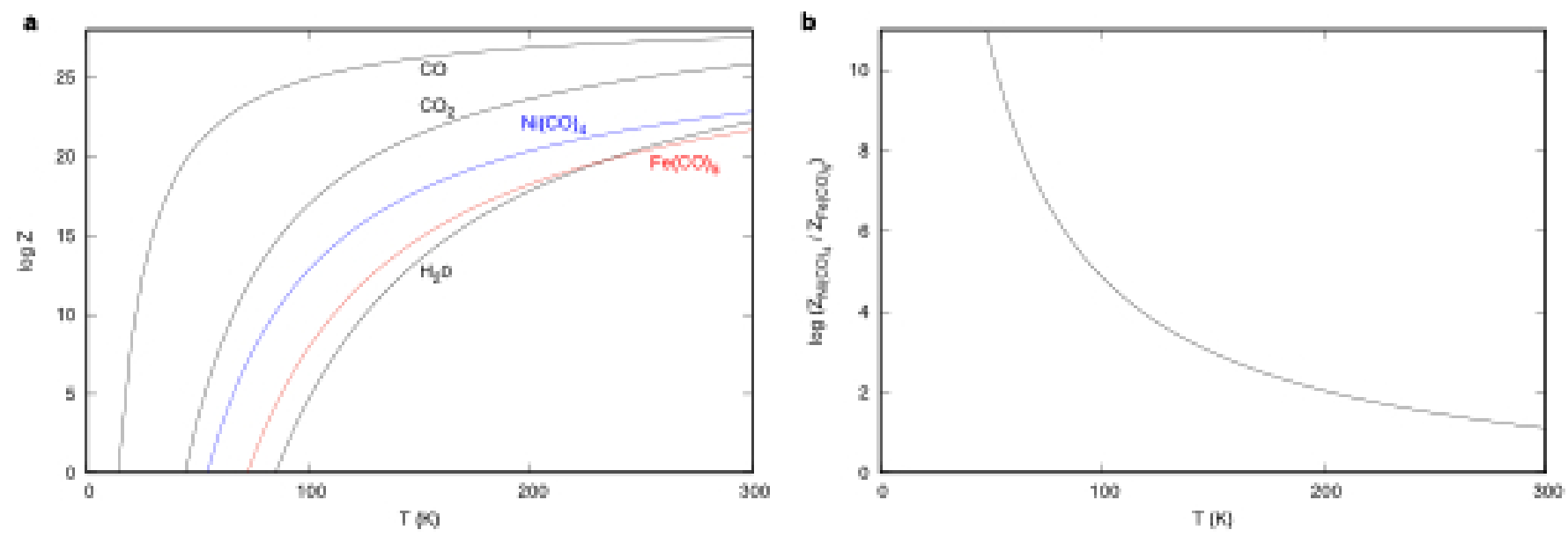

Extended Data Fig. 3. Iron and nickel carbonyl sublimation properties. a, The sublimation rates ( $Z$ in molecules $\mathrm{cm}^{-2} \mathrm{~s}^{-1}$ ) of $\mathrm{Fe}$ and $\mathrm{Ni}$ carbonyls as a function of temperature, compared to those of the main ices in comets. The carbonyl rates are intermediate between those of $\mathrm{H}_{2} \mathrm{O}$ and $\mathrm{CO}_{2}$. b. The sublimation rate ratio $\mathrm{Ni}(\mathrm{CO})_{4}$ over $\mathrm{Fe}(\mathrm{CO})_{5}$ shows that the sublimation rate of $\mathrm{Ni}(\mathrm{CO})_{4}$ is significantly higher than the one of $\mathrm{Fe}(\mathrm{CO})_{5}$. These quantities were computed as follows. As ${ }^{52,53}$, we estimate the condensation or sublimation temperature $T_{s}$ of these compounds by solving the equation $f_{x} n k T_{s}=P_{v, x}\left(T_{s}\right)$ where $f_{x}$ is the relative abundance of species $x, n$ the number density of the gas, $k$ the Boltzmann constant, and $P_{v, x}$ the vapor pressure given by the relation $\log P_{v, x}(T)=$ $-A / T+B$. The constants $A$ and $B$ for $\mathrm{Fe}(\mathrm{CO})_{5}$ and $\mathrm{Ni}(\mathrm{CO})_{4}$ are obtained from ${ }^{54,55}$, that is $A=2097 \mathrm{~K}$ and $B=11.62$ for $\mathrm{Fe}(\mathrm{CO})_{5}, A=1534 \mathrm{~K}$ and $B=10.87$ for $\mathrm{Ni}(\mathrm{CO})_{4}$, with $P_{v, x}$ in dyn $\mathrm{cm}^{-2}$. We consider relative abundances $f_{x}$ between $10^{-3}$ and $10^{-5} \times f_{x}\left(\mathrm{H}_{2} \mathrm{O}\right)$, for both $\mathrm{Fe}(\mathrm{CO})_{5}$ and $\mathrm{Ni}(\mathrm{CO})_{4}$, and we adopt $n=10^{13} \mathrm{~cm}^{-3} \mathrm{as}^{53}$. The resulting sublimation temperatures of the iron and nickel carbonyls (respectively $97-108 \mathrm{~K}$ and $74-82 \mathrm{~K}$, depending on $f_{x}$ ) are intermediate between the sublimation temperatures of $\mathrm{H}_{2} \mathrm{O}$ and $\mathrm{CO}_{2}(152 \mathrm{~K}$ and $72 \mathrm{~K})$, while $\mathrm{CO}$ sublimates at $25 \mathrm{~K}^{53}$. The sublimation rate (in molecules $\mathrm{cm}^{-2} \mathrm{~s}^{-1}$ ) from the surface of a pure ice into vacuum can be expressed as ${ }^{56}: Z_{x}(T)$ $=P_{v, x}(T)\left(2 \pi m_{x} k T\right)^{-1 / 2}$ where $T$ is the ice temperature and $m_{x}$ the mass of the species $x$.

\section{End Notes}

Supplementary Information is available for this paper.

\section{Data availability statement}

The datasets analyzed during the current study are available at the ESO Science Archive Facility at http://archive.eso.org/eso/eso_archive_main.html, under programmes 073.C-0525, 075.C- 0355(A), 080.C-0615, 086.C-0958, 087.C-0929, 270.C-5043, 274.C-5015, 2100.C-5035(A), 280.C-5053 and 2101.C-5051. Correspondence and requests for materials should be addressed to J. Manfroid.

\section{Email and orcid :}

J. Manfroid jmanfroid@gmail.com 0000-0002-6930-2205 
Hutsemékers d.hutsemekers@uliege.be

Jehin ejehin@uliege.be 0000-0001-8923-488X

Reprints and permissions information is available at www.nature.com/reprints

\section{Author contribution}

$\mathrm{JM}$ analyzed the spectra and the coma profiles and wrote the main text. DH contributed to the proposals and observations, reduced and calibrated the spectra, built the fluorescence model, computed the carbonyl sublimation properties, and wrote the supplementary information. EJ lead the UVES proposals and made most of the observations. All authors contributed to the discussion and the final text.

\section{Acknowledgements}

We thank Dr. P. van Hoof for useful discussions on the iron atomic data and their uncertainties. We thank R. Hewins and Prof. R. Warin for discussions about various Fe and Ni rich compounds in meteorites. We thank C. Arpigny, D. Bockelée-Morvan, A. Decock, C. Opitom, H. Rauer, P. Rousselot, and B. Yang for leading some UVES proposals, and the ESO staff for service mode observations. JM, DH, and EJ are honorary Research Director, Research Director and Senior Research Associate at the F.R.S-FNRS, respectively.

Competing interests. The authors declare no competing interests. 


\title{
Supplementary information Iron and nickel atoms in comet atmospheres
}

\author{
J. Manfroid, D. Hutsemékers, and E. Jehin \\ STAR Institute, University of Liège \\ Allée du 6 Août 19c, B-4000 Liège, Belgium
}

\section{Contents}

1 FeI and NiI fluorescence models and abundance measurements 1

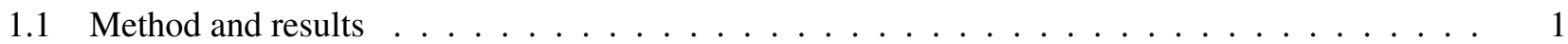

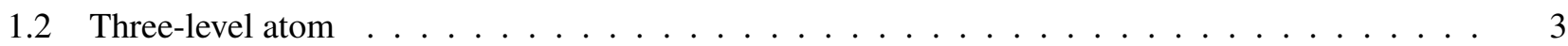

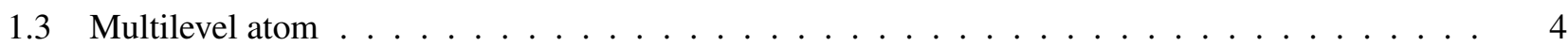

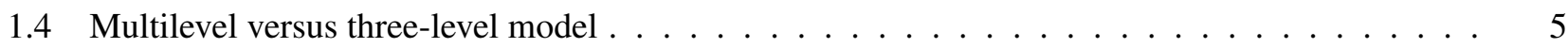

1.5 Tests, possible improvements, and robustness of the results . . . . . . . . . . . 5

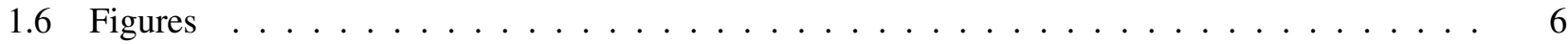

\section{FeI and NiI fluorescence models and abundance measurements}

\subsection{Method and results}

Preston [1] found that the intensity of the FeI and NiI emission lines observed in comet C/1965 S1 (Ikeya-Seki) at $0.14 \mathrm{au}$ from the Sun can be related to the energy of the upper level of the transitions through a Boltzmann distribution, and that these lines are likely formed by resonance fluorescence. A simple "curve-of-growth" analysis then provided the excitation temperature of the lines and allowed the determination of the NiI/FeI abundance ratio. In Sect. 1.2, we explicitly reformulate the resonance fluorescence model [2, 3] considering a 3-level atom and assuming that the solar radiation can be represented by a diluted blackbody. For each comet the excitation temperature is empirically determined following Eq. 14 using the observed FeI emission line intensities and atomic data from the Atomic Line List v2.05 [4]. Fig. S1 illustrates the empirical method used to estimate the excitation temperature for two representative comets. For NiI, the smaller range of upper energy levels precludes an accurate determination of the excitation temperature but, given the similar atomic level structure of FeI and NiI, we assume $T(\mathrm{NiI})=T(\mathrm{FeI})$ as in [1-3]. Suspected blends ${ }^{1}$ are not considered and a few recurrent outliers are discarded from the analysis, in particular a few FeI lines with lower energy levels higher than $2 \mathrm{eV}$, and the two NiI lines with the smallest $\log (g f)$. In two comets, the number of observed FeI lines is too small to derive the temperature and we adopt $T=4000 \pm 1000 \mathrm{~K}$, which is representative of the sample. The NiI/FeI abundance ratio is finally obtained from Eq. 15 with $\log U_{\mathrm{Ni}} / U_{\mathrm{Fe}}=0.06 \pm 0.02$ computed for the temperature range $3500-5000 \mathrm{~K}$. Errors on the mean abundance ratio account for the dispersion of the $C$ values (fixed to 0.3 dex when the line number is smaller than 3 ) and the range of acceptable temperatures. The NiI/FeI abundance ratios computed with the 3-level model are given in Extended Data Table $2^{2}$. The metallic lines found in comet Ikeya-Seki were similarly analyzed ${ }^{3}$, considering only the FeI and NiI lines that are also detected in our spectra to avoid any bias. The

\footnotetext{
${ }^{1}$ Examples are the bright FeI $3859.91 \AA$ and $3856.37 \AA$ lines which are blended with the generally much stronger CN R4 $3859.95 \AA$ and R9 $3856.40 \AA$ A lines, respectively, as well as the NiI $3458.46 \AA$ line which is often overwhelmed by some underlying line.

${ }^{2}$ Abundance ratios show little dependence on the temperature as far as the same excitation temperature is used for both FeI and NiI. Column densities, on the other hand, strongly depend on the adopted temperature and are therefore not reported in the Table.

${ }^{3}$ The line intensities are taken from [1]. This paper provides equivalent widths in units of the sky spectrum intensity and argues that the sky spectrum is mostly independent of wavelength ("white") so that the equivalent widths can be used as intensities with an error that can be as high as $30 \%$.
} 
$\mathrm{NiI} / \mathrm{FeI}$ abundance ratio we derive for comet Ikeya-Seki is in excellent agreement with previous studies [1-3]. Although the 3-level model seems to provide a reasonably good interpretation of the FeI and NiI emission spectrum, it is based on approximations that need to be tested, in particular the assumption of identical excitation temperatures for the FeI and NiI lines, and the use of a blackbody for the solar radiation (strong metallic lines in absorption are known to sprinkle the solar spectrum). We therefore built a multilevel atomic model where the true solar spectrum is taken into account. The model is detailed in Sect. 1.3. Input atomic data are extracted from the Atomic Line List v2.05 [4]. For FeI, we consider transitions with lower levels in the energy range 0-20000 $\mathrm{cm}^{-1}$, upper levels in the energy range $0-40000 \mathrm{~cm}^{-1}$, and line strengths with $A_{k i}>10^{3} \mathrm{~s}^{-1}$. These constraints ensure that all useful observed transitions are included, while keeping the number of levels reasonably small. This results in 427 transitions with wavelengths between $2500 \AA$ and $13000 \AA$ for 85 energy levels including the ground level. For NiI, we use the same constraints with upper levels in the energy range $0-50000 \mathrm{~cm}^{-1}$. For the solar spectrum, we use the calibrated high-resolution spectrum of [5]. The spectral range is $2960-13000 \AA$, with a resolving power between 350000 (UV) and 500000 (IR). For the spectral range 2000-2960 , we use the calibrated solar spectrum of [6] which has a lower spectral resolution of $0.1 \AA$.

In Fig. S2, the FeI lines intensities computed with the model are compared to the measured intensities in four comets. The computed spectra reproduce reasonably well the observed spectra, although for some lines, differences can be significant, possibly due to unrecognized blends, measurement errors, or uncertainties of the atomic data which can be large [7]. For comets 103P and especially C/2016 R2, the dispersion of the intensity ratios is higher than for Ikeya-Seki, and some bright lines not well reproduced. Differences between Ikeya-Seki and other comets with respect to the model could be, at least in part, attributed to the strong difference in heliocentric velocities, $+110 \mathrm{~km} \mathrm{~s}^{-1}$ for Ikeya-Seki versus $+3.5,-5.6$ and $+25.6 \mathrm{~km} \mathrm{~s}^{-1}$ for 103P, C/2016 R2 and C/2002 T7, respectively. The solar spectrum shows deep FeI and NiI absorption lines so that the radiation reaching the comet is strongly dimmed at low heliocentric velocities (Fig. S3). For high enough Doppler velocities, the radiation reaching the comet is expected to be more homogeneous for the different metallic lines, and closer to a blackbody. This emphasizes the importance of the comet heliocentric velocity as the cometary FeI and NiI transitions can sample very different portions of the solar spectrum depending on the Doppler shift.

In Fig. S4, the $I_{\mathrm{obs}} / I_{\text {mod }}$ ratios (Eq. 20) are shown on a logarithmic scale for both the FeI and NiI lines. While the dispersion of $\log \left(I_{\mathrm{obs}} / I_{\text {mod }}\right)$ can be high, significant differences between the relative mean FeI and NiI column densities are clearly seen between these comets. The column densities of FeI and NiI are given in Extended Data Table 2, as well as the derived NiI/FeI abundance ratios. The errors of the mean column densities are estimated from the standard deviation of the logarithmic column densities computed from individual lines (fixed to 0.3 dex when the line number is smaller than 3 ), divided by the square root of the number of lines. Errors thus include measurement errors and uncertainties of the atomic data.

In Sect. 1.4, we compare the results from the two models. The fact that the excitation temperatures estimated with the 3-level model are systematically lower than the Sun color temperature can be explained, at least in part, by the presence of strong absorption lines in the true solar spectrum. The NiI/FeI abundance ratios computed with the 3-level model nicely correlate with those ones derived from the multilevel model although they show a systematic shift of $\sim 0.2$ dex (Fig. S6). The difference between the two models appears mainly due a proper account of the solar radiation spectrum in the multilevel model rather than to the number of levels used in the computation.

Tests and possible improvements are discussed in Sect. 1.5. They essentially show that the measured abundance ratios are robust. In particular, increasing the number of atomic transitions and levels does not improve the derived quantities. To improve the fits, better intensity measurements (deblending) and more accurate atomic data would be needed. Finally one should keep in mind that the fit of individual lines may also be affected by the lower spectral resolution of the solar spectrum at wavelengths smaller than $2960 \AA$. Several transitions from the ground level do occur in that wavelength range for which the incoming solar flux might not be accurately estimated, especially at low heliocentric velocities. 


\subsection{Three-level atom}

In addition to the ground level, FeI and NiI show a few metastable lower levels and upper levels of opposite parity. Observed transitions occur between the two sets of levels [2,3,8]. We then consider a 3-level atom, excited by resonance fluorescence. Statistical equilibrium for the ground and the lower levels writes

$$
\begin{aligned}
n_{l}\left(A_{l g}+B_{l g} J_{l g}\right)+n_{u}\left(A_{u g}+B_{u g} J_{u g}\right) & =n_{g}\left(B_{g l} J_{g l}+B_{g u} J_{g u}\right), \\
n_{g}\left(B_{g l} J_{g l}\right)+n_{u}\left(A_{u l}+B_{u l} J_{u l}\right) & =n_{l}\left(A_{l g}+B_{l g} J_{l g}+B_{l u} J_{l u}\right),
\end{aligned}
$$

where $n_{g}, n_{l}$, and $n_{u}$ are the volume density of atoms in the ground, lower, and upper levels, respectively. $A, B$ are the Einstein coefficients and $J$ the mean intensity of the radiation. $J_{i j}=\int J(v) \phi(v) d v$ over the natural line profile that is $J_{i j} \simeq J\left(v_{i j}\right)$ with $\int \phi(v) d v=1$. Since the lower level is metastable, transitions $g \leftrightarrow l$ can be neglected and these relations simplify to

$$
\begin{aligned}
\frac{n_{u}}{n_{g}} & =\frac{B_{g u} J_{g u}}{\left(A_{u g}+B_{u g} J_{u g}\right)}, \\
\frac{n_{u}}{n_{l}} & =\frac{B_{l u} J_{l u}}{\left(A_{u l}+B_{u l} J_{u l}\right)} .
\end{aligned}
$$

The Einstein coefficients are related by $A_{u i} / B_{u i}=2 h v_{u i}^{3} / c^{2}$ and $g_{i} B_{i u}=g_{u} B_{u i}$ for $i=g, l$. Assuming $J_{v}=W B_{v}$ where $B_{v}(T)=\left(2 h v^{3} / c^{2}\right) /\left(e^{h v / k T}-1\right)$ represents the solar blackbody radiation at frequency $v$ and temperature $T$, and $W$ the dilution factor

$$
W=\frac{1}{2}\left(1-\sqrt{1-\frac{R_{\odot}^{2}}{r^{2}}}\right) \simeq \frac{1}{4} \frac{R_{\odot}^{2}}{r^{2}},
$$

$R_{\odot}$ denoting the solar radius and $r$ the comet heliocentric distance, we derive

$$
\frac{n_{u}}{n_{i}}=\frac{g_{u}}{g_{i}} \frac{1}{1+\left(e^{h v_{i u} / k T}-1\right) / W} .
$$

Since $W \ll 1$ and assuming $h v \gg k T$ (Wien approximation) we can finally write

$$
\begin{aligned}
n_{u} / n_{g} & =W\left(g_{u} / g_{g}\right) 10^{-\theta \chi_{u}}, \\
n_{u} / n_{l} & =W\left(g_{u} / g_{l}\right) 10^{-\theta\left(\chi_{u}-\chi_{l}\right)}, \\
n_{l} / n_{g} & =\left(g_{l} / g_{g}\right) 10^{-\theta \chi_{l}},
\end{aligned}
$$

where $\theta=5040 \mathrm{~K} / T$ and $\chi_{u}\left(\chi_{l}\right)$ is the energy of the upper (lower) level in eV. If $n$ is the volume density of the atom in all states, $n_{l} / n=g_{l} 10^{-\theta \chi_{l}} / U(T)$ where $U(T)$ is the partition function, so that we have

$$
\frac{n_{u}}{n}=W \frac{g_{u} 10^{-\theta \chi_{u}}}{U(T)}
$$

Due to the dilution of the exciting radiation, the upper levels are weakly populated with respect to the lower ones and $U(T) \simeq g_{g}+\sum_{l} g_{l} 10^{-\theta \chi_{l}}$.

The intensity integrated over the line of sight of the radiation emitted in the transition $u \rightarrow i$ with $i=g$, $l$, is given by

$$
I_{v}=\int j_{v} d s \quad \text { where } \quad j_{v}=\frac{1}{4 \pi} n_{u} A_{u i} h v_{u i} \phi(v) .
$$

Using the oscillator strength

$$
f_{i u}=\frac{m c^{3}}{8 \pi^{2} e^{2} v_{u i}^{2}} \frac{g_{u}}{g_{i}} A_{u i}
$$


we write

$$
I_{v}=N W \frac{8 \pi^{2} e^{2} h}{4 \pi m} \frac{g_{i} f_{i u}}{\lambda_{u i}^{3}} \frac{10^{-\theta \chi_{u}}}{U(T)} \phi(v)
$$

where $N=\int n d s$ is the column density. This relation can be written in the form

$$
\log \frac{I \lambda^{3}}{g f}=-\theta \chi_{u}+C
$$

that allows the empirical determination of $\theta$ by plotting $\log \left(I \lambda^{3} / g f\right)$ against $\chi_{u}$ for observed spectral lines. $I=\int I_{\nu} d v=\int I_{\lambda} d \lambda$ is the intensity (surface brightness) integrated over the observed line profile. Relative abundances of two atoms 1 and 2 can then be obtained using

$$
\log \frac{N_{1}}{N_{2}}=\left(C_{1}-C_{2}\right)+\log \frac{U_{1}}{U_{2}},
$$

where the constants $C$ are computed from Eq. 14 for each observed line of a given atom using the estimated value of $\theta$, and then averaged over all observed lines.

\subsection{Multilevel atom}

We consider $m$ energy levels. Statistical equilibrium for level $i$ reads

$$
\sum_{\substack{j=1 \\ j \neq i}}^{m} n_{j} Q_{j i}=n_{i} \sum_{\substack{j=1 \\ j \neq i}}^{m} Q_{i j}
$$

where $Q_{i j}=B_{i j} J_{i j}$ for $i<j, Q_{i j}=A_{i j}+B_{i j} J_{i j}$ and $A_{i j}=B_{i j}\left(2 h v_{i j}^{3} / c^{2}\right)$ for $i>j$, and $g_{i} B_{i j}=g_{j} B_{j i}$. By defining $Q_{i i}=-\sum_{\substack{j=1 \\ j \neq i}}^{m} Q_{i j}$ and $n_{j}^{\prime}=n_{j} / n_{1}$, we finally have for level $i$

$$
\sum_{j=2}^{m} n_{j}^{\prime} Q_{j i}=-Q_{1 i}
$$

that is a system of $m$ linear equations with $m-1$ unknowns. By dropping the $i=1$ redundant equation, and denoting $\alpha_{i j}=Q_{j+1, i+1}, \beta_{i}=Q_{1, i+1}$ and $x_{j}=n_{j+1}^{\prime}$ for $i, j=1, m-1$, we write the matrix equation

$$
\left(\begin{array}{cccc}
\alpha_{11} & \alpha_{12} & \cdots & \alpha_{m-1,1} \\
\alpha_{21} & \alpha_{22} & \cdots & \alpha_{m-1,2} \\
\vdots & \vdots & \ddots & \vdots \\
\alpha_{m-1,1} & \alpha_{m-1,2} & \cdots & \alpha_{m-1, m-1}
\end{array}\right)\left(\begin{array}{c}
x_{1} \\
x_{2} \\
\vdots \\
x_{m-1}
\end{array}\right)=\left(\begin{array}{c}
\beta_{1} \\
\beta_{2} \\
\vdots \\
\beta_{m-1}
\end{array}\right)
$$

that we solve with the Gauss-Jordan elimination method [9] to derive the atomic level population ratios $n_{j}^{\prime}$. With $\left.n_{j} / n=n_{j}^{\prime} /\left(1+\sum_{k=2}^{m} n_{k}^{\prime}\right)\right)$, line intensities are finally obtained for each transition $j \rightarrow i$ :

$$
I_{j i}=\frac{h}{4 \pi} N \frac{n_{j}^{\prime}}{1+\sum_{k=2}^{m} n_{k}^{\prime}} A_{j i} v_{j i} .
$$

The high-resolution solar spectral irradiance $F_{\lambda}$ given in [5] and [6] at 1 au is converted into mean intensity in the coma using $J_{i j}=J\left(v_{i j}\right)=(4 \pi c)^{-1} F_{\lambda} \lambda_{i j}^{2} r^{-2}$ where $r$ is in au.

Denoting $I_{\text {mod }}\left(=I_{j i}\right.$ for $\left.N=1 \mathrm{~cm}^{-2}\right)$ and $I_{\mathrm{obs}}$ the computed and observed line intensities integrated over the 
observed line profile, respectively, the logarithmic column density is derived using

$$
\log N=\log \frac{I_{\mathrm{obs}}}{I_{\mathrm{mod}}}
$$

For a set of observed lines, $\log N$ is averaged over all lines, and the abundance ratio of atoms 1 and 2 given by the ratio of their mean column densities.

\subsection{Multilevel versus three-level model}

The comparison of the two models (Fig. S5) shows that the 3-level atomic populations reproduce fairly well the populations computed with the multilevel model, provided that the blackbody temperature adopted in the 3-level model is significantly lower than the Sun color temperature. We also estimated the excitation temperature using in Eq. 14 the FeI line intensities generated by the multilevel model. For cometary heliocentric velocities of $5 \mathrm{~km} \mathrm{~s}^{-1}$ and $100 \mathrm{~km} \mathrm{~s}^{-1}$, we derive $T \simeq 4000 \mathrm{~K}$ and $T \simeq 4500 \mathrm{~K}$, respectively. The fact that the excitation temperature is smaller than the color temperature of the Sun $(\sim 5800 \mathrm{~K})$ is thus mostly due to the fact that the flux from the solar spectrum is fainter than a blackbody due to the strong absorption lines, in particular when the Doppler velocity is close to zero (Fig. S3).

As shown in Fig. S6, there is an overall agreement between the NiI/FeI abundances estimated with the two models but also a clear systematic offset: the abundance ratios derived with the 3-level model are about 0.2 dex larger than the abundance ratios derived with the multilevel model. A better agreement is obtained by assuming $T(\mathrm{NiI})=T(\mathrm{FeI})+180 \mathrm{~K}$ in the 3-level model.

\subsection{Tests, possible improvements, and robustness of the results}

We performed several tests modifying the constraints on the selected energy levels and minimum transition strength, and considering up to 668 energy levels and 3435 transitions for FeI. No significant changes of the level populations or line intensities were observed in the spectral range of interest, i.e., 3300-4500 $\AA$. We also considered alternative atomic data sets, in particular from [10], and found no significant differences in the model results.

The effect of collisions was also investigated, with the aim to better fit the observed line intensities. A collisional term $C_{i j}$ was added to the $Q_{i j}$ defined in Sect. 1.3, with $C_{i j} / C_{j i}=\left(g_{j} / g_{i}\right) \times 10^{\theta\left(\chi_{i}-\chi_{j}\right)}$. An approximate formula for estimating collisions with electrons is given by [11]. Given the low density and low temperature prevailing in cometary comae, collisions with electrons are negligible. Collisions with atoms or molecules might be considered but, as far as we know, no estimates are available for cometary atmospheres. We thus parametrized collisional de-excitation as $C_{j i}=\epsilon\left[\theta\left(\chi_{j}-\chi_{i}\right)\right]^{\delta}$ where $\epsilon$ and $\delta$ are free parameters. Tests done by varying these parameters indicate that introducing collisions does not improve the model and most often gives poorer fits.

We also considered extinction by dust in the inner coma using the standard CCM extinction curve [12]. Both the incoming solar radiation and the outward emitted lines were assumed to be reddened. For C/2016 R2, assuming $A_{V} \simeq 1.5$ (which is a reasonable value for the extinction in the inner coma [13]) makes the dispersion in Fig. S4 slightly smaller, while keeping the abundance ratio unchanged. 


\subsection{Figures}
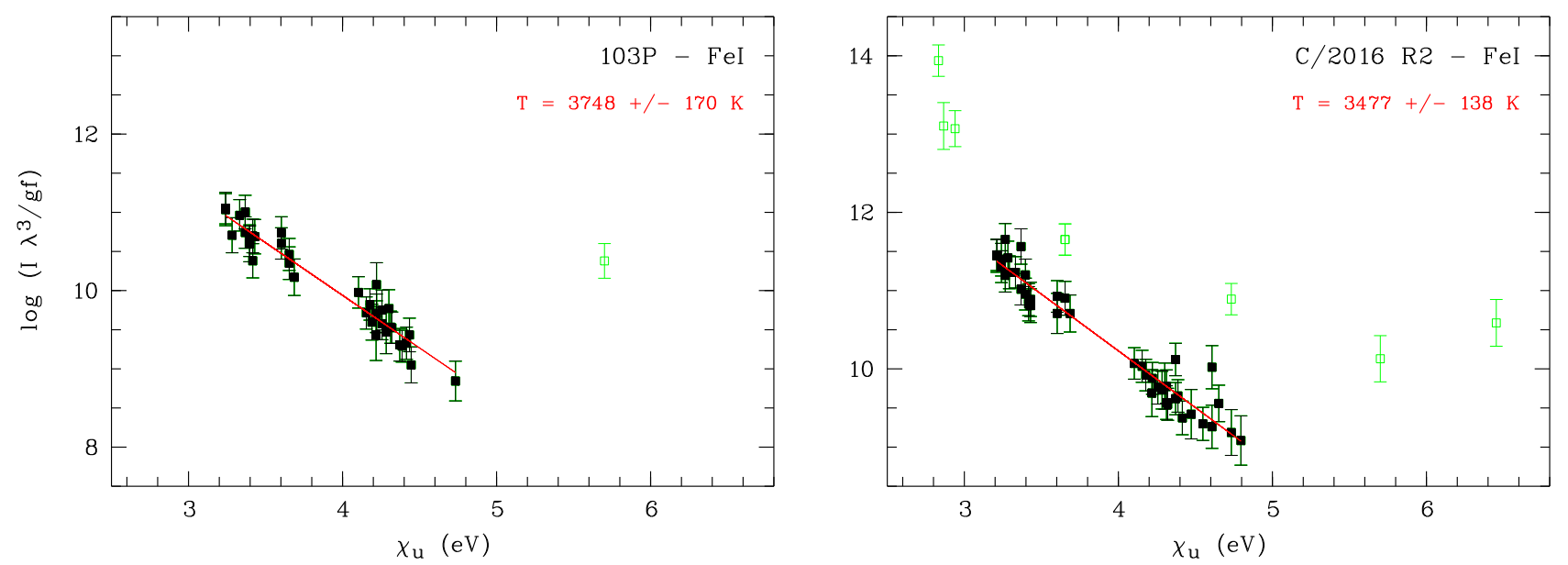

Figure S1: Empirical determination of the excitation temperature. FeI emission lines observed in two representative comets are used. Outliers not considered in the fit are shown in green. For C/2016 R2 (and only for that comet) additional outliers were discarded through an iterative fit. Error bars account for the errors on the measured intensities and an uncertainty of $\log (g f)$ assumed to be 0.2 dex. 

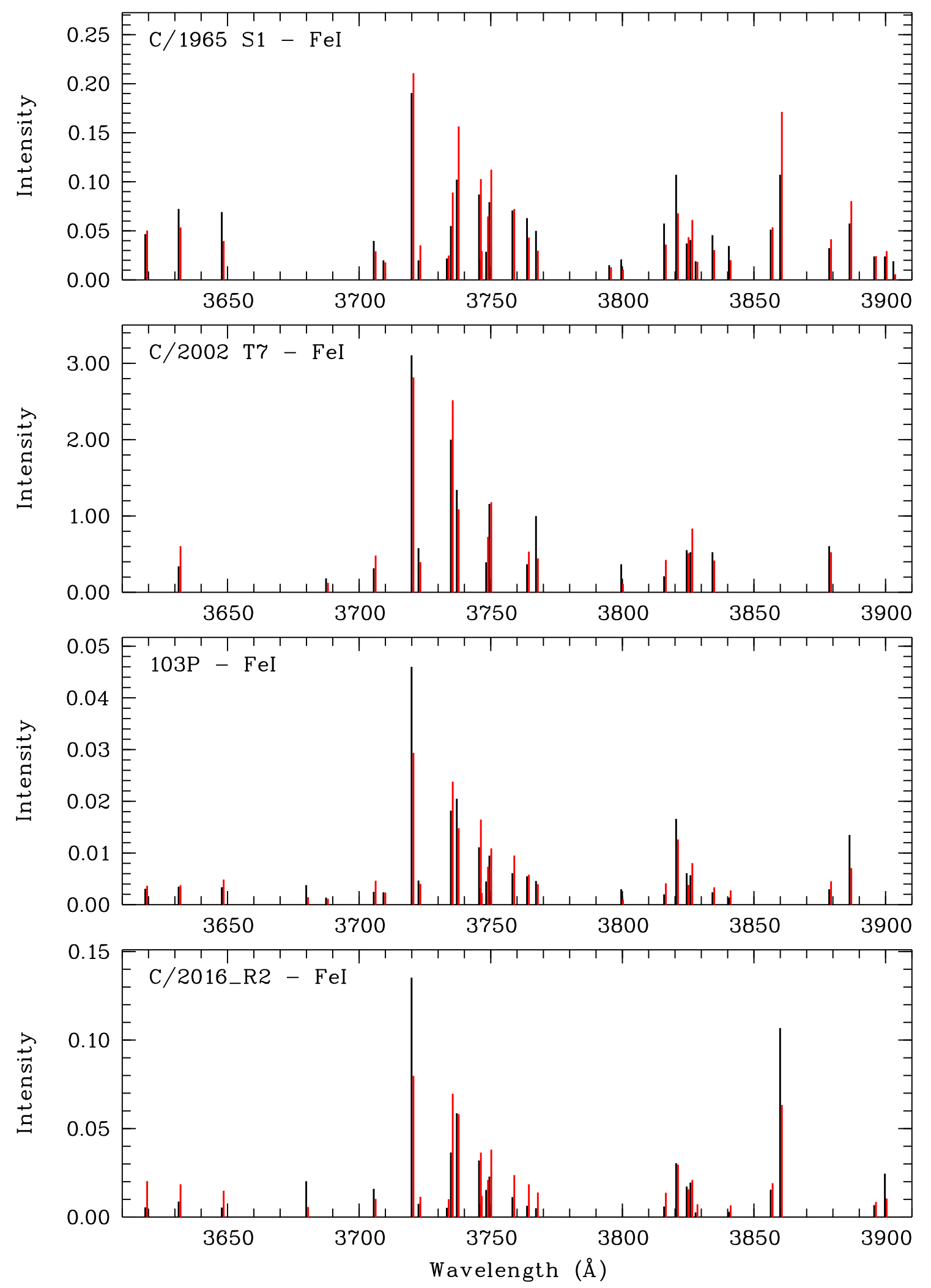

Figure S2: Comparison of the observed and modeled spectra. A portion of the FeI spectrum is shown for four representative comets. Observed line intensities are in black; computed line intensities are in red and shifted by $0.7 \AA$ for visibility. Intensities are in arbitrary units. The intensities from the model have been scaled using the ratio $I_{\mathrm{obs}} / I_{\mathrm{mod}}$ averaged over all observed lines. 

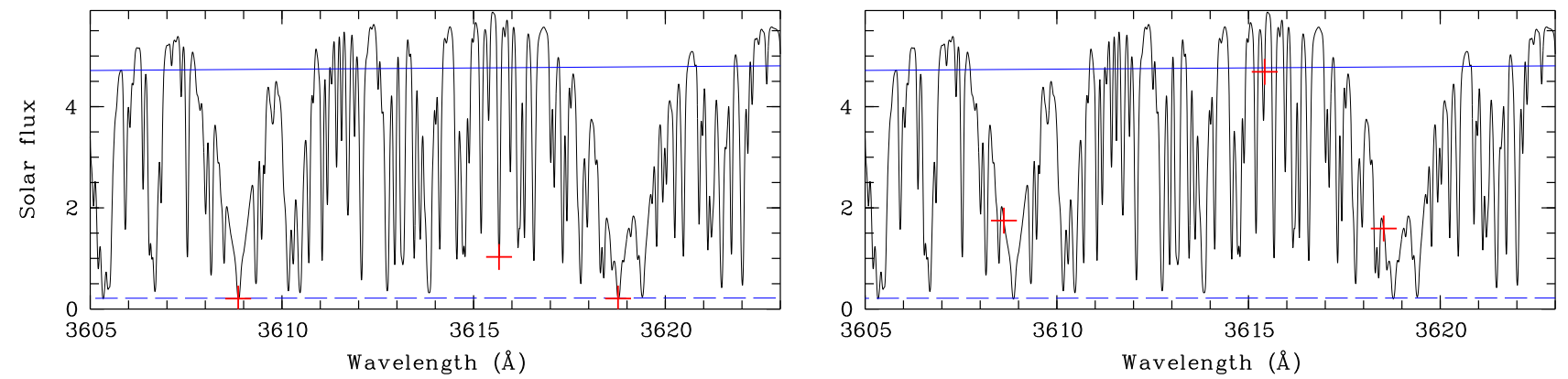

Figure S3: Sample of the Kurucz solar spectrum. The red crosses indicate the value of the solar flux involved in three cometary FeI transitions. The solid blue line represents a blackbody spectrum with $T=5800 \mathrm{~K}$, and the dashed blue line a blackbody spectrum with $T=4000 \mathrm{~K}$. Cometary heliocentric velocities are equal to $0 \mathrm{~km} \mathrm{~s}^{-1}$ (left) and $+20 \mathrm{~km} \mathrm{~s}^{-1}$ (right). The solar flux is given in arbitrary units.
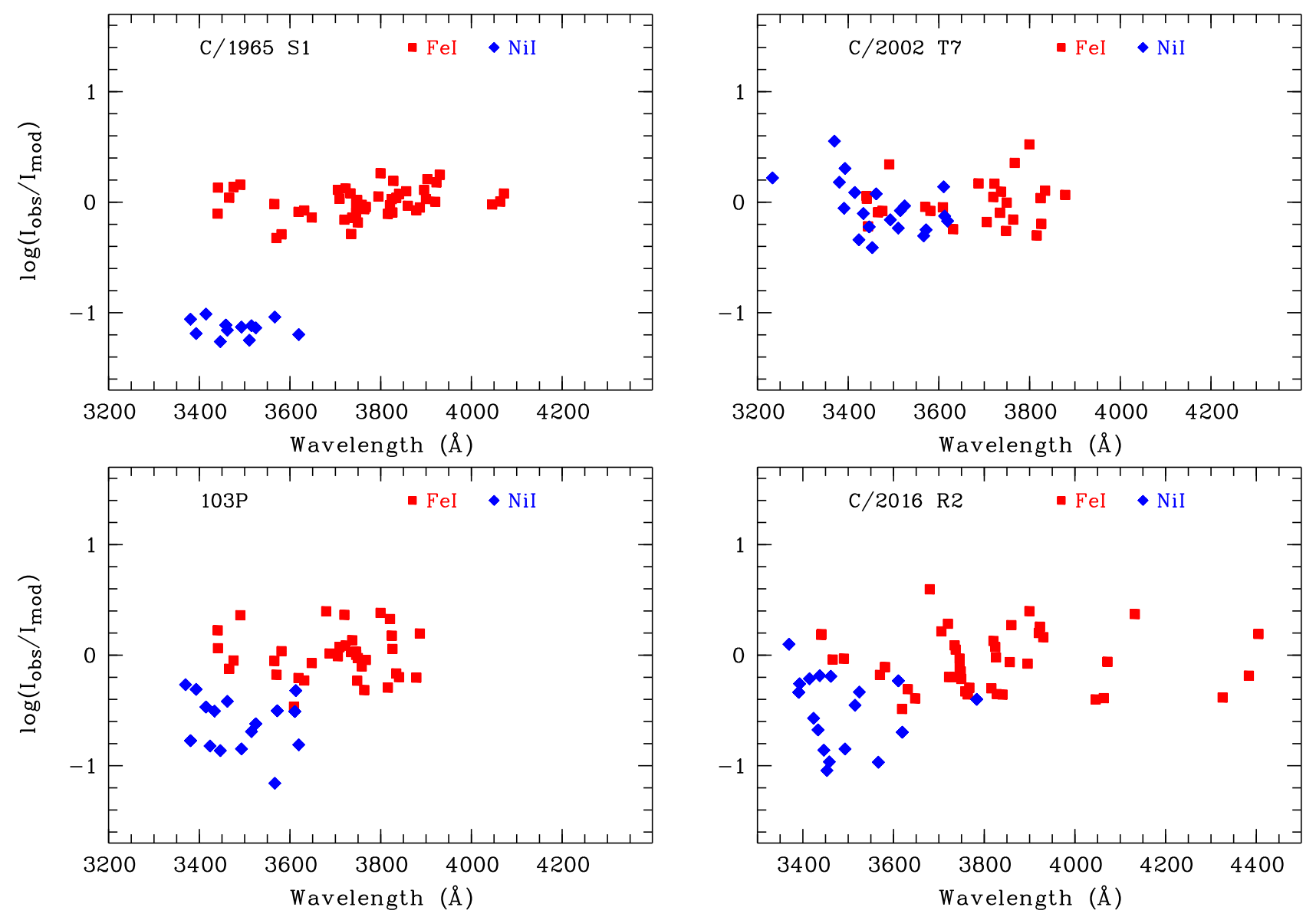

Figure S4: Abundance ratio determination. The ratio $\log \left(I_{\mathrm{obs}} / I_{\mathrm{mod}}\right)=\log N$ measured for the FeI (red) and NiI (blue) lines in four comets. The ratios have been shifted on the y-axis so that the mean of $\log \left(I_{\mathrm{obs}} / I_{\bmod }\right)$ is zero for FeI. The few outliers have been discarded. The difference of the means computed for all FeI and NiI lines gives the $\log (\mathrm{NiI} / \mathrm{FeI})$ abundance ratio. 

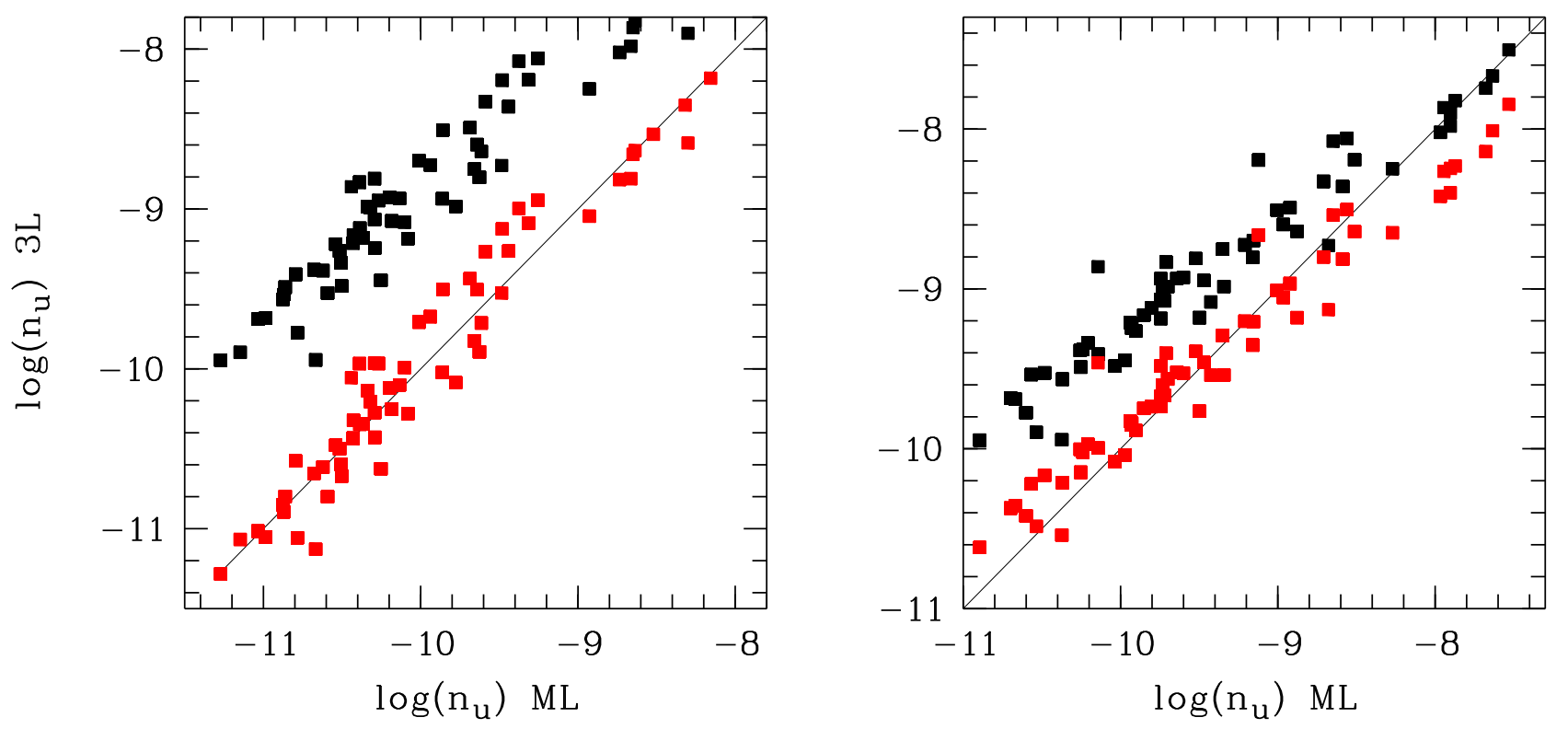

Figure S5: Comparison of upper level populations. The level populations are computed for FeI with the 3-level (3L) and multilevel (ML) atomic models. Left: Black squares represent 3-level populations computed with a blackbody temperature of $5800 \mathrm{~K}$ (the color temperature of the Sun) while red squares represent 3-level populations computed with a temperature of $4400 \mathrm{~K}$ in better agreement with the multilevel populations computed with the Kurucz solar spectrum. Right: Same as in the previous figure, but with the solar spectrum shifted by $20 \mathrm{~km} \mathrm{~s}^{-1}$. The red squares represent 3-level populations computed with a temperature of $5000 \mathrm{~K}$.
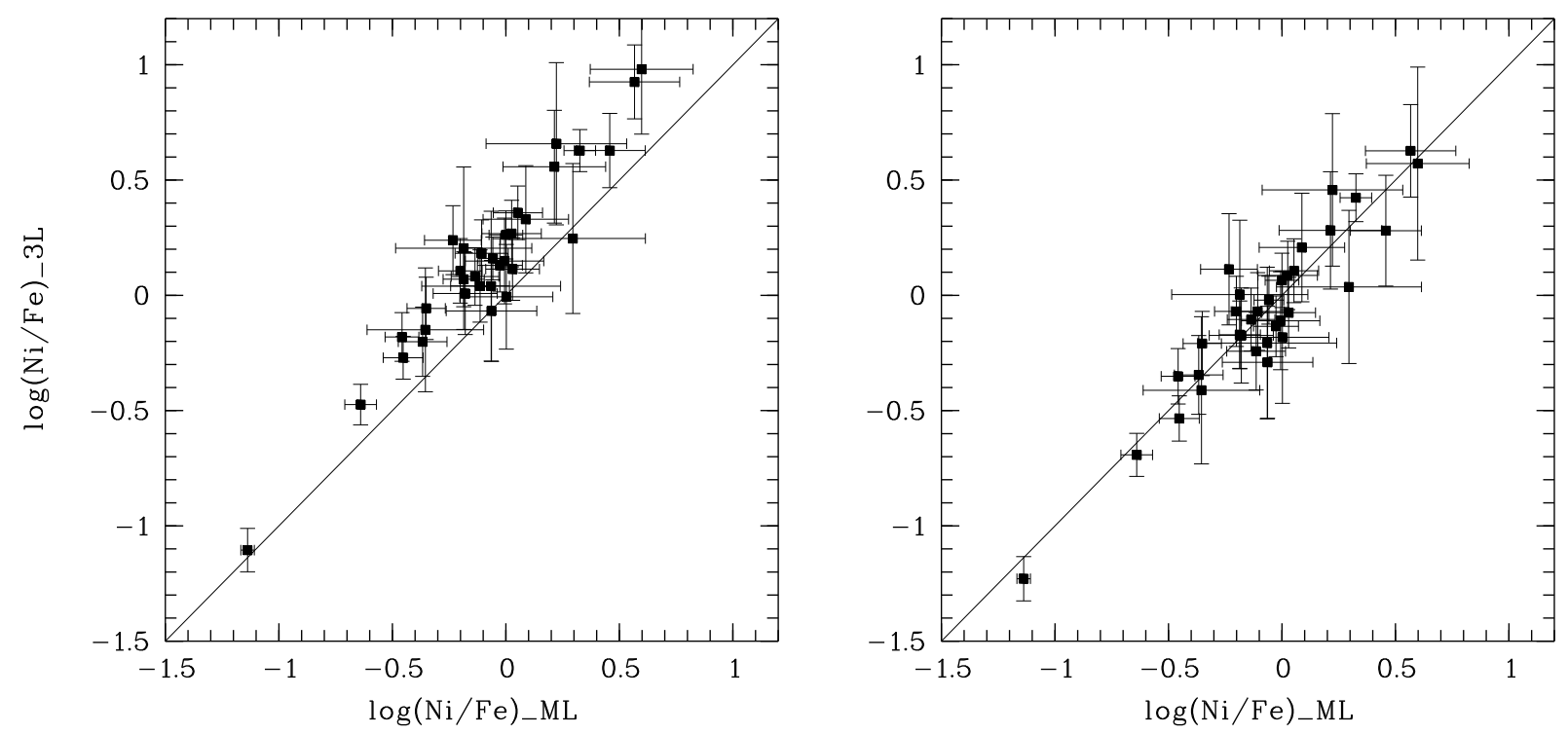

Figure S6: Abundances from the two models. Comparison of $\log (\mathrm{NiI} / \mathrm{FeI})$ abundances derived with the multilevel (ML) model and the 3-level (3L) model for the comets of our sample. Left: Assuming $T(\mathrm{NiI})=T(\mathrm{FeI})$. Right: With $T(\mathrm{NiI})=T(\mathrm{FeI})+180 \mathrm{~K}$. 


\section{References}

${ }^{1}$ G. W. Preston, “The spectrum of Ikeya-Seki (1965f)”, ApJ 147, 718-742 (1967).

${ }^{2}$ C. Arpigny, "On the nature of comets.", in Proceedings of the robert a. welch foundation conferences on chemical research xxi, cosmochemistry, edited by W. Mulligan (1978), p. 9.

${ }^{3}$ C. Arpigny, "Relative abundances of the heavy elements in comet Ikeya-Seki /1965 VIII/", in Liege international astrophysical colloquia, Vol. 22, edited by A. Boury, N. Grevesse, and L. Remy-Battiau, Liege International Astrophysical Colloquia (Jan. 1979), pp. 189-197.

${ }^{4}$ P. A. M. van Hoof, "Recent Development of the Atomic Line List", Galaxies 6, 63 (2018).

${ }^{5}$ R. L. Kurucz, I. Furenlid, J. Brault, and L. Testerman, Solar flux atlas from 296 to $1300 \mathrm{~nm}$ (1984).

${ }^{6}$ L. A. Hall and G. P. Anderson, "High-resolution solar spectrum between 2000 and 3100 Å.”, J. Geophys. Res. 96, 12, 927-12, 931 (1991).

${ }^{7}$ J. R. Fuhr and W. L. Wiese, "A Critical Compilation of Atomic Transition Probabilities for Neutral and Singly Ionized Iron”, Journal of Physical and Chemical Reference Data 35, 1669-1809 (2006).

${ }^{8}$ C. E. Moore, Partial Grotriam diagrams of astrophysical interest (1968).

${ }^{9}$ W. H. Press, S. A. Teukolsky, W. T. Vetterling, and B. P. Flannery, Numerical recipes in FORTRAN. The art of scientific computing (1992).

${ }^{10}$ G. Nave, S. Johansson, R. C. M. Learner, A. P. Thorne, and J. W. Brault, “A New Multiplet Table for Fe i”, ApJS 94, 221 (1994).

${ }^{11}$ H. van Regemorter, "Rate of Collisional Excitation in Stellar Atmospheres.", ApJ 136, 906 (1962).

${ }^{12}$ J. A. Cardelli, G. C. Clayton, and J. S. Mathis, "The Relationship between Infrared, Optical, and Ultraviolet Extinction", ApJ 345, 245 (1989).

${ }^{13}$ P. Lacerda and D. Jewitt, "Extinction in the Coma of Comet 17P/Holmes", ApJ 760, L2, L2 (2012). 